\title{
Wave potential and the one-dimensional windkessel as a wave-based paradigm of diastolic arterial hemodynamics
}

\author{
Jonathan P. Mynard ${ }^{1,2}$ \\ Joseph J. Smolich ${ }^{1,2}$ \\ ${ }^{1}$ Heart Research Group, Murdoch Childrens Research Institute, Parkville, VIC, Australia \\ ${ }^{2}$ Department of Paediatrics, University of Melbourne, Parkville, VIC, Australia
}

Abbreviated Title: Wave potential and the one-dimensional windkessel

\section{Author Contributions:}

JPM: conception and design, modelling, data analysis \& interpretation, manuscript preparation JJS: experimental studies, manuscript revisions

Both authors approved the final manuscript.

\section{Address for Correspondence:}

Jonathan P. Mynard

Heart Research, Clinical Sciences

Murdoch Childrens Research Institute,

Flemington Road, Parkville, VIC, 3052, Australia

Phone: 61-3-9936-6038

E-mail: jonathan.mynard@mcri.edu.au 


\section{Abstract}

Controversy exists about whether one-dimensional (1D) wave theory can explain the 'selfcancelling' waves that accompany the diastolic pressure decay and discharge of the arterial reservoir. Although it has been proposed that reservoir and wave effects be treated as separate phenomena, thus avoiding the issue of self-cancelling waves, we have argued that reservoir effects are a phenomenological and mathematical subset of wave effects. However, a complete wave-based explanation of self-cancelling diastolic expansion (pressure-decreasing) waves has not yet been advanced. These waves are present in the forward and backward components of arterial pressure and flow $\left(P_{ \pm}\right.$and $Q_{ \pm}$), which are calculated by integrating incremental pressure/flow changes $\left(d P_{ \pm}\right.$and $\left.d Q_{ \pm}\right)$. While the integration constants for this calculation have previously been considered arbitrary, we show that physiologically meaningful constants can be obtained by identifying 'undisturbed pressure' as mean circulatory pressure. Using a series of numerical experiments, absolute $P_{ \pm}$and $Q_{ \pm}$values are shown to represent 'wave potential', gradients of which produce propagating wavefronts. With the aid of a ' $1 \mathrm{D}$ windkessel', we show how wave theory predicts discharge of the arterial reservoir. Simulated data, along with hemodynamic recordings in seven sheep, suggest that self-cancelling diastolic waves arise from repeated and diffuse reflection of the late systolic forward expansion wave throughout the arterial system and at the closed aortic valve, along with progressive leakage of wave potential from the conduit arteries. The combination of wave and wave potential concepts leads to a comprehensive one-dimensional (i.e. wave-based) explanation of arterial hemodynamics, including the diastolic pressure decay.

Keywords: wave separation, arterial hemodynamics, reservoir, one-dimensional model 


\section{Introduction}

Separation of arterial pressure and flow signals into forward $\left(P_{+}\right.$and $\left.Q_{+}\right)$and backward $\left(P_{-}\right.$and $Q_{-}$) wave components provides important information about interactions between the ventricle and vascular network $(17,18,35,51)$. Although the gold-standard for assessing wave dynamics over the past 40 years, wave separation has been the subject of recent controversy. At issue is that, during diastole when there is no aortic inflow, wave separation predicts that $P_{+}$and $P_{-}$are near-equal, while $Q_{+}$and $Q_{-}$are equal in magnitude but opposite in sign, the latter giving rise to the term 'self-cancelling' waves $(5,48,49)$.

On one side of the debate, self-cancelling waves have been deemed an "implausible artefact" of the wave separation technique, given an expectation that forward wave components should be zero when the aortic valve is closed (5). Moreover, wave separation applied to a beat with a long diastole results in a cross-over and progressive increase in the magnitude of flow components as diastole progresses (Fig. 1), a puzzling feature that is difficult to explain physiologically. To resolve these problems, Wang et al (49) proposed the reservoir-wave paradigm, in which a zerodimensional (0D) 'reservoir pressure' is first subtracted from measured pressure. When subjected to wave separation, the remaining one-dimensional (1D) 'excess pressure' does not exhibit self-cancelling waves. On the basis of this finding, proponents of this paradigm have concluded that phenomena related to arterial reservoir filling and discharge, such as the diastolic pressure decay, lie outside the explanatory scope of 1D wave theory (5).

The reservoir-wave paradigm has, however, also attracted criticism $(24,30-32,43,44,52)$ because, while it appears to resolve problems with diastolic wave separation, the accompanying pattern of systolic wave reflection is fundamentally different to widely-held concepts derived from a large body of experimental and clinical evidence $(31,32)$. Indeed, in numerical experiments of vascular networks containing reflection sites with known impedance mismatches, and thus reflection coefficients, wave separation using excess pressure underestimated or entirely 
missed positive wave reflection, overestimated negative wave reflection or produced spurious waves not associated with any impedance mismatches. These problems were not encountered with traditional wave separation, which accurately predicted the character and magnitude of reflected waves $(24,31)$.

If traditional wave separation is to remain a gold-standard approach for evaluating wave interactions, however, a wave-based explanation is required for self-cancelling diastolic waves and the diastolic pressure decay. The precursors to such an explanation have been provided by three observations. First, the realization that the $0 \mathrm{D}$ windkessel model, which is often used to explain reservoir phenomena, is a mathematical reduction of the $1 \mathrm{D}$ wave equations $(21,31,43)$ implies that reservoir effects are a subset of wave effects and hence that self-cancelling waves are not an artefact. Second, the existence of equal pressure and opposing flow wave components is to be expected if a backward-running wave encounters a closed aortic valve with a reflection coefficient close to unity $(30,52)$. Third, the recognition that arterial wave reflection occurs in a distributed nature and involves many reflections and re-reflections, as well as wave trapping (1, $2,4)$, together with the finding that the systolic rise in reservoir pressure arises from peripherally reflected systolic waves (1), is suggestive of a relationship between wave reflection and reservoir phenomena.

Accordingly, the aim of the current study was to develop a wave-based paradigm of diastolic arterial hemodynamics that explains self-cancelling waves and reservoir phenomena such as the diastolic pressure decay. The study consisted of two complementary components. A theoretical framework for the paradigm was first developed, with numerical experiments used to illustrate the key concepts. The paradigm was then evaluated with in vivo experiments in which hemodynamics were altered with vasoactive drugs and aortic constriction. Our approach involves a subtle but important refinement of traditional wave separation, namely the definition of non-arbitrary integration constants when calculating $P_{ \pm}$and $Q_{ \pm}$. By reconsidering the concept of undisturbed pressure (19), this step adds a new dimension to the standard interpretation of 
wave separation in that the integration constants, and hence the absolute values of $P_{ \pm}$and $Q_{ \pm}$, are bestowed with physical meaning via a concept termed 'wave potential'.

\section{Theoretical development}

\section{Computational modelling methods}

The basic computational methods for the numerical experiments have been described in detail previously. Briefly, the non-linear one-dimensional form of the Navier-Stokes equations were solved as in (29) along with a physiologically realistic non-linear pressure-area relation (27). Reflection coefficient boundary conditions and, where applicable, an elastance ventricle inlet boundary condition were implemented using methods described in $(25,28,29)$.

\section{Wave separation with arbitrary integration constants}

In linear wave separation $(17,35,51)$, incremental changes in the forward $(+)$ and backward $(-)$ components of pressure $(P)$ and flow $(Q)$ are additive (Fig. 1), i.e.

$$
\begin{gathered}
d P=d P_{+}+d P_{-} \\
d Q=d Q_{+}+d Q_{-}
\end{gathered}
$$

Defining characteristic impedance as $Z_{c}=\rho c / A$, where $\rho$ is blood density, $c$ is wave speed and $A$ is vessel cross-sectional area, the water hammer equation states that

$$
d P_{ \pm}= \pm Z_{c} d Q_{ \pm}
$$

Combining Equations 1 and 2 with Equation 3, it can be shown that

$$
\begin{gathered}
d P_{ \pm}=\frac{1}{2}\left(d P \pm Z_{c} d Q\right) \\
d Q_{ \pm}=\frac{1}{2}\left(d Q \pm \frac{1}{Z_{c}} d P\right)
\end{gathered}
$$

Integration of these equations leads to the forward and backward wave components of $P$ and $Q$,

$$
P_{ \pm}=\frac{1}{2}\left[\left(P-P^{0}\right) \pm Z_{c}\left(Q-Q^{0}\right)\right]+P_{ \pm}^{0}
$$




$$
Q_{ \pm}=\frac{1}{2}\left[\left(Q-Q^{0}\right) \pm \frac{1}{Z_{c}}\left(P-P^{0}\right)\right]+Q_{ \pm}^{0}
$$

Here $P^{0}$ and $Q^{0}$ are initial values of $P$ and $Q$, while $P_{ \pm}^{0}$ and $Q_{ \pm}^{0}$ (the integration constants) are initial values of $P_{ \pm}$and $Q_{ \pm}$. Since wave components have previously been considered pertinent only to the pulsatile aspects of pressure/flow waveforms, $P_{ \pm}^{0}$ and $Q_{ \pm}^{0}$ have been chosen arbitrarily. For graphing convenience (as in Fig. 1), $P_{+}^{0}$ and $P_{-}^{0}$ are often set to end-diastolic pressure $\left(P_{\text {ed }}\right)(11,14,18)$. Alternatively, some have chosen $P_{ \pm}^{0}=0.5 P_{\text {ed }}(36)$ or have assigned $P_{+}=P_{\text {ed }}$ and $P_{-}=0(15,20,37)$; in both cases, $P=P_{+}+P_{-}$. Others have set mean $P_{+}$and $P_{-}$to zero (3) as an expression of the arbitrariness of the mean value, while still others have avoided specifying absolute values of $P_{ \pm}$altogether $(16,22,51)$.

In most papers, $Q_{+}^{0}$ and $Q_{-}^{0}$ have been set to zero or end-diastolic flow, as in Fig 1 . This approach seems reasonable for steady-state beats since $Q_{+}$and $Q_{-}$, although equal and opposite during diastole, approach zero at the start of the next beat. However, two potential problems are apparent with this approach. First, if $Q_{+}$and $Q_{-}$approach zero at the start of the next beat, this implies that the time it takes for all waves in the arterial system (generated by the heart and by reflection in the vasculature) to fully dissipate is coincidentally and precisely one heart period.

A second problem is that if regularity of the cardiac cycle is perturbed, then it is evident that arterial waves are not fully dissipated in one heart period. This may be seen with two examples. First, in an ectopic beat containing a long diastole, $Q_{+}$and $Q_{-}$cross over and progressively increase in magnitude after $P$ falls below $P_{\text {ed }}$ (Fig. 1). In addition, Fig. 2A shows ascending aortic $P$ and $Q$ in an experimental study where wave separation was performed on multiple beats before and during euthanasia. Interestingly, although $Q$ falls to zero and $P$ asymptotes to a constant value after $\sim 10 \mathrm{sec}$, the wave components $\left(P_{ \pm}\right.$and $\left.Q_{ \pm}\right)$do not asymptote towards zero, as would be expected. Particularly difficult to explain physiologically are the large positive and 
negative asymptotic values of $Q_{-}$and $Q_{+}$respectively. This observation suggests that the choice $Q_{ \pm}^{0}=0$ is not physiological and also implies that $Q_{ \pm}^{0}$ should not be considered arbitrary, since intuitively $Q_{ \pm}$should asymptote to zero after a long period of asystole; similar arguments apply to $P_{ \pm}^{0}$

These observations lead to two important questions. First, can non-arbitrary values of the integration constants be defined that result in physiologically meaningful results during an extended asystole? Second, if non-arbitrary constants are used, what are the implications for interpreting diastolic wave components and reservoir discharge? Before addressing these questions, we must first reconsider the role of undisturbed pressure in wave separation.

\section{Undisturbed pressure $\left(P_{\mathrm{ud}}\right)$}

In his foundational book "Waves in Fluids", Lighthill defined waves as being driven by an 'excess pressure' above an 'undisturbed pressure' $\left(P_{\mathrm{ud}}\right)$, the latter being an equilibrium pressure distribution that does not contain pressure gradients (apart from hydrostatic gradients) or produce fluid accelerations (19). Under the assumption that the arterial circulation is a system in steadystate oscillation, pressure and flow are often treated as the sum of a mean value and a series of sinusoidal (Fourier) harmonics. On this view, mean pressure is unrelated to waves, "the steady flow term and average pressure term are disregarded" (51) when performing wave analysis, and therefore mean pressure is identified as $P_{\text {ud }}$. Similarly, in time-domain wave separation, $P_{\text {ed }}$ is usually designated as the undisturbed reference point, a choice that also treats the arterial system as being in steady-state oscillation.

The concept of arterial steady-state oscillation has been questioned by Parker (34) and Hughes et al (12), however, who pointed out that although the arterial system is normally subject to periodic forces (ventricular ejection), pressure rapidly decays if even a single heart beat is missed. Conversely, when cardiac contraction recommences after a long period of asystole, mean pressure rises as pressure/flow pulses are forced into the system until, after some time, an 
equilibrium is reached $(2,33)$. If heart rate increases/decreases, then (all else being equal) mean pressure rises/falls. Mean pressure therefore does not exist physiologically and is only a manifestation of the size and frequency of the input forcing waves, along with wave reflections and the rate at which the arterial reservoir drains during diastole. As expressed by Hughes et al, “where, if not from waves generated by the heart does mean pressure ultimately derive?" (12).

Thus, although arterial pressure and flow can be treated mathematically as periodic, from a mechanistic point of view, mean $P$ and $P_{\text {ed }}$ arise from waves. If neither mean $P$ nor $P_{\text {ed }}$ should be identified as $P_{\text {ud }}$, how then should $P_{\text {ud }}$ be defined?

\section{Wave separation with non-arbitrary integration constants}

The chief insight from Fig. $2 \mathrm{~A}$ is that to achieve physiologically intuitive $P_{ \pm}$and $Q_{ \pm}$during a period of asystole, we require that $P_{ \pm}^{\infty}=0$ and $Q_{ \pm}^{\infty}=0$ ( $\infty$ here means 'after a long period of asystole'). This restriction implies that $P_{\mathrm{ud}}$ should be identified as mean circulatory pressure $\left(P_{\mathrm{mc}}\right)$, i.e. the pressure that prevails when no pressure gradients exist in the system (8). Consider an undisturbed hemodynamic state in which there is no potential energy for blood flow anywhere in the circulation. In such a state, $Q=0$ everywhere and, ignoring hydrostatic gradients, pressure is everywhere equal to $P_{\text {ud }}$. Assuming that $P_{ \pm}=0$ and $Q_{ \pm}=0$ when $P=P_{\text {ud }}$, and assuming $Z_{\mathrm{c}}$ is constant, from Equations 6 and 7 it can be shown that

$$
\begin{gathered}
P_{ \pm}^{0}=\frac{1}{2}\left[P^{0}-P_{\text {ud }} \pm Z_{c} Q^{0}\right] \\
Q_{ \pm}^{0}=\frac{1}{2}\left[Q^{0} \pm \frac{1}{Z_{c}}\left(P^{0}-P_{\text {ud }}\right)\right]
\end{gathered}
$$

The separated $P$ and $Q$ components, incorporating these non-arbitrary integration constants, are then

$$
P_{ \pm}=\frac{1}{2}\left[P-P_{\text {ud }} \pm Z_{c} Q\right]
$$




$$
Q_{ \pm}=\frac{1}{2}\left[Q \pm \frac{1}{Z_{c}}\left(P-P_{\text {ud }}\right)\right]
$$

Measured $P$ and $Q$ can then be considered to consist of the following components,

$$
P=P_{\text {ud }}+P_{+}+P_{-}
$$

where $P_{+}+P_{-}$is excess pressure (19), and

$$
Q=Q_{+}+Q_{-}
$$

Importantly, noting that $P_{\text {ud }} \ll \operatorname{mean}(P)$, Equation 12 expresses the view that waves predominantly determine mean pressure. Fig. $2 \mathrm{~B}$ shows that if we set $P_{\mathrm{ud}}=P_{\mathrm{mc}}$ in Equations 10 and $11, P_{ \pm}$and $Q_{ \pm}$now asymptote to zero after a long period of asystole.

\section{Wave potential}

Having addressed the problem of non-zero $P_{ \pm}^{\infty}$ and $Q_{ \pm}^{\infty}$ through a physiologically justifiable definition of 'undisturbed state', Fig. 3 reveals a new issue requiring consideration, namely, that during steady state beats the mean value of $Q_{+}$(in this example, $13.7 \mathrm{~L} / \mathrm{min}$ ) is far greater than mean $Q(2.8 \mathrm{~L} / \mathrm{min})$, while mean $Q_{-}$is substantial and negative $(-11.0 \mathrm{~L} / \mathrm{min})$. On first glance, these steady-state values of $Q_{ \pm}$may appear non-physiological. To explain why these values do have physiological meaning, consider what would happen if, during a normal hemodynamic steady-state, the aorta was suddenly occluded in two locations. Assuming no side branches lay between the two occlusion sites, there would be no flow in or out of the occluded segment and pressure would be constant $\left(P_{\text {constant }}\right)$, with a similar value to that prevailing before the occlusions were applied (i.e. $P_{\text {constant }}>P_{\text {ud }}$ ). Shortly after the occlusions commence, no propagating waves or inflow/outflow will exist, hence $d P_{ \pm}=0, d Q_{ \pm}=0, Q=0$ and $P=P_{\text {constant }}$. However, Equations 10 and 11 suggest that the wave components of pressure and flow are non-zero as follows,

$$
P_{ \pm}=\frac{1}{2}\left[P_{\text {constant }}-P_{\text {ud }}\right]
$$




$$
Q_{ \pm}= \pm \frac{1}{2}\left[\frac{1}{Z_{c}}\left(P_{\text {constant }}-P_{\text {ud }}\right)\right]
$$

For example, if $P_{\text {ud }}=0, P_{\text {constant }}=100 \mathrm{mmHg}$ and $Z_{c}=0.05 \mathrm{mmHg} . \mathrm{s} / \mathrm{mL}$ (typical values for a human ascending aorta), then $P_{ \pm}=50 \mathrm{mmHg}$ and $Q_{ \pm}= \pm 60 \mathrm{~L} / \mathrm{min}$. The physical relevance of these values, while difficult to appreciate while the occlusions continue, immediately becomes apparent when the occlusions are released.

To illustrate, Fig. 4 shows a numerical experiment in which two tubes are connected at $x=0$, with a barrier between the two tubes removed at $t=10 \mathrm{~ms}$. Before the barrier is released (Fig. 4, $t=0)$, pressure in tube $1(x<0)$ is $P_{\text {constant }}=10 \mathrm{mmHg}$, while in tube $2(x>0)$ pressure is $P_{\text {ud }}=0$. After removal of the barrier, two pressure-flow waves propagate in opposite directions (Fig. 4, $t=15 \mathrm{~ms}$ and $t=30 \mathrm{~ms}$ ). As would be expected, these waves decrease pressure in tube 1 and increase pressure in tube 2 (thereby equalizing pressure at the junction), while both waves cause flow to increase in the positive $x$ direction.

Where do these waves come from and could their pressure/flow effects have been predicted? Noting that $Z_{c}=0.064 \mathrm{mmHg} . \mathrm{s} / \mathrm{mL}$ in both tubes, according to Equations 14 and $15 P_{ \pm, 1}=5$ $\mathrm{mmHg}$ and $Q_{ \pm, 1}= \pm 78.6 \mathrm{~mL} / \mathrm{s}$ at $t=0$ in tube 1 and $P_{ \pm, 2}=Q_{ \pm, 2}=0$ in tube 2 . The pressure/flow wave component differences at $x=0$ are therefore

$$
\begin{aligned}
\Delta P_{+} & =P_{+, 1}-P_{+, 2}=5 \mathrm{mmHg} \\
\Delta P_{-} & =P_{-, 2}-P_{-, 1}=-5 \mathrm{mmHg} \\
\Delta Q_{+} & =Q_{+, 1}-Q_{+, 2}=78.6 \mathrm{~mL} / \mathrm{s} \\
\Delta Q_{-} & =Q_{-, 2}-Q_{-, 1}=78.6 \mathrm{~mL} / \mathrm{s}
\end{aligned}
$$

Hence, Equations 14 and 15 exactly predict the magnitude of the pressure/flow wavefronts in Fig. 4. Mean or absolute values of $P_{ \pm}$and $Q_{ \pm}$may therefore be interpreted as pressure/flow wave potential, with any spatial gradient in this potential giving rise to propagating pressure/flow wavefronts. 


\section{Waves and arterial reservoir/windkessel function}

Now consider what occurs as time progresses in the numerical experiment depicted in Fig. 4, in which tube 1 has a closed end at $x=-15 \mathrm{~cm}$ (analogous to a closed aortic valve) whereas tube 2 is infinitely long. The backward-travelling wave (seen at $t=15,30 \mathrm{~ms}$ ) in tube 1 decreases pressure from $P^{0}(10 \mathrm{mmHg})$ to $0.5 P^{0}$ but increases forward flow. When this wave reaches the closed end, it undergoes complete reflection and propagates forwards, decreasing pressure further from $0.5 P^{0}$ to $P_{\text {ud }}$ (zero) and decreasing forward flow to zero ( $t=50 \mathrm{~ms}$ ). Hence the effect of this wave is to empty the fluid reservoir that is tube 1. Assuming a constant compliance $C$ (i.e. linear pressure-area relation) over the pressure range $P_{\text {ud }}$ to $P^{0}$, the volume $(V)$ emptied is

$$
V=-\left(P^{0}-P_{\text {ud }}\right) C
$$

where the negative sign indicates a volume decrease. Furthermore, the time it takes the wave to traverse the length $(L)$ of tube 1 is $L / c$, where $c$ is the wave speed. The pressure-decreasing backward-running expansion wave (BEW) initiates reservoir emptying and the reflected forward-running expansion wave (FEW) terminates the emptying. Emptying therefore occurs at the rate $Q_{-}^{0}$ over the time period $2 L / c$. The emptying volume can also be calculated via the time integral of flow as

$$
V=\frac{2 L}{c} Q_{-}^{0}
$$

Hence, higher absolute values of $Q_{ \pm}$signify both greater wave potential and greater fluid volume discharge potential, consistent with Equation 11 which shows that $Q_{ \pm}$is inversely proportional to $Z_{\mathrm{c}}$. For a given pressure above $P_{\text {ud }}$, a stiffer or smaller vessel (with higher $Z_{\mathrm{c}}$ or $c$ ) will store less volume and have lower values of $Q_{ \pm}$. Conversely, for a given $Z_{\mathrm{c}}$ or $c$, a higher pressure 
implies greater volume storage and is revealed as larger absolute $Q_{ \pm}$values. These principles are demonstrated using numerical simulations in Fig. 5.

\section{Self-cancelling waves}

A wave-based explanation of the diastolic pressure decay and the 'self-cancelling' expansion waves is now within reach. Consider a second numerical experiment, depicted in the lower panel of Fig. 6. The first tube (length $60 \mathrm{~cm}$, area $5.0 \mathrm{~cm}^{2}$, wave speed $4.0 \mathrm{~m} / \mathrm{s}, Z_{c}=0.064$ mmHg.s/mL, closed inlet) represents the low impedance large arteries during diastole and the second tube (area $1.59 \mathrm{~cm}^{2}$, wave speed $20.0 \mathrm{~m} / \mathrm{s}, Z_{c}=1.0 \mathrm{mmHg} . \mathrm{s} / \mathrm{mL}$, well-matched outlet to simulate an infinitely long tube) represents the high impedance small arteries. Initially, flow is zero everywhere and pressure is $P_{1}^{0}=80 \mathrm{mmHg}$ in the large tube and $P_{2}^{0}=0 \mathrm{mmHg}$ in the small tube. Fig. 6 shows the evolution of pressure and flow at three locations in the large tube. Due to the initial pressure difference (and hence wave potential) between the two tubes, a BEW arises in the large tube and causes a step increase in flow $\left(\Delta Q_{-, 1}=81.2 \mathrm{~mL} / \mathrm{s}\right)$ and a step decrease in pressure $\left(\Delta P_{-, 1}=-5.2 \mathrm{mmHg}\right)$. Generalising Equations 16 and 17 to the situation where a barrier is removed between two tubes with differing characteristic impedance $\left(Z_{1}\right.$ and $\left.Z_{2}\right)$ and initial pressure $\left(P_{1}^{0}\right.$ and $\left.P_{2}^{0}\right)$, it can be shown, using Equations 3, 10 and 11 and by assuming continuity of pressure and flow at the junction of the two tubes, that the resultant wavefronts are

$$
\begin{gathered}
\Delta P_{+, 2}=\left(P_{+, 2}-P_{+, 1}\right) T_{12}^{P}=\left(P_{1}^{0}-P_{2}^{0}\right) \frac{Y_{1}}{Y_{1}+Y_{2}} \\
\Delta P_{-, 1}=\left(P_{-, 1}-P_{-, 2}\right) T_{21}^{P}=\left(P_{2}^{0}-P_{1}^{0}\right) \frac{Y_{2}}{Y_{1}+Y_{2}} \\
\Delta Q_{+, 2}=\left(Q_{+, 1}-Q_{+, 2}\right) T_{12}^{Q}=\left(P_{1}^{0}-P_{2}^{0}\right) \frac{Y_{1} Y_{2}}{Y_{1}+Y_{2}} \\
\Delta Q_{-, 1}=\left(Q_{-, 2}-Q_{-, 1}\right) T_{21}^{Q}=\left(P_{1}^{0}-P_{2}^{0}\right) \frac{Y_{1} Y_{2}}{Y_{1}+Y_{2}}
\end{gathered}
$$


where $T_{i j}^{P}=2 Y_{i} /\left(Y_{i}+Y_{j}\right)$ and $T_{i j}^{Q}=2 Y_{j} /\left(Y_{i}+Y_{j}\right)$ are the respective pressure and flow transmission coefficients from tube $i$ to tube $j$ (25), expressed in terms of characteristic $\operatorname{admittances}\left(Y_{1}=1 / Z_{1}\right.$ and $\left.Y_{2}=1 / Z_{2}\right)$.

As shown in Fig. 6, the initial pressure-decreasing BEW propagates backwards and is completely reflected at the closed aortic valve, giving rise to a FEW that further decreases pressure. When this FEW reaches the junction of the two tubes, it is partially reflected according to the reflection coefficient

$$
\Gamma=\left(Y_{1}-Y_{2}\right) /\left(Y_{1}+Y_{2}\right)
$$

In this example, $\Gamma=0.88$ and therefore most of the wave energy is reflected, giving rise to a second BEW slightly smaller than the first. This wave is again reflected completely at the aortic valve, partially reflected again at the tube junction and so on (Fig. 6). After the wave has propagated back and forth $n$ times, pressure at the outlet of the large tube is

$$
P(n)=P^{0}-2 \Delta P \frac{1-\Gamma^{n}}{1-\Gamma}
$$

which is a discrete exponential equation. An equivalent continuous equation is

$$
P(t)=P^{0} \mathrm{e}^{-b t}
$$

where $b=-\ln (\Gamma) / t_{T}$ and $t_{T}$ is the transit time of the large tube $(0.15 \mathrm{~s})$. Hence, we see that an exponential pressure decay arises from progressive 'leakage' of wave potential from a low impedance to a high impedance vascular domain through repeated reflection and re-reflection of expansion waves.

The windkessel description of the pressure decay can be derived from this wave description as follows. Noting that $\ln \Gamma \approx \Gamma-1$ (from the Taylor series expansion), we have

$$
b \approx-\frac{\Gamma-1}{t_{T}}
$$

Since $Y_{1} \gg Y_{2}$, Equation 22 can also be approximated as $\Gamma \approx\left(Y_{1}-Y_{2}\right) / Y_{1}$. It follows that 


$$
b \approx \frac{Y_{2}}{Y_{1} t_{T}}
$$

If $Y_{2}$ is identified as the reciprocal of peripheral resistance (i.e. $Y_{2}=1 / R_{p}$ ), transit time as $t_{T}=L / c$ (where $L$ and $c$ are vessel length and wave speed), and $Y_{1}=A /(\rho c)$, then

$$
b \approx \frac{\rho c^{2}}{A L R_{p}}=\frac{1}{R_{p} C}=\frac{1}{\tau}
$$

where $C=A L /\left(\rho c^{2}\right)$ is large vessel compliance and $\tau=R_{p} C$ is the familiar time constant of the exponential pressure decay in the 2-element windkessel. The two-tube model shown in Fig. 6 may therefore be described as a 'one-dimensional windkessel'.

\section{Origin of diastolic expansion waves}

The physiological system differs from the conceptual model in the previous section in that 1) there is no well-defined interface between large and small arteries, and 2) there is no barrier between large and small arteries that is suddenly removed to generate the initial BEW. From where then do the diastolic expansion waves originate? During diastole, the aortic valve is closed and therefore the FEW component must arise from complete reflection of the BEW component. However, what gives rise to the BEW component? There are two possible explanations. First, this wave component could arise from negative reflection of the early systolic forward compression wave (FCW). However, this is unlikely since the systemic arteries tend to produce positive wave reflection (32); moreover, the self-cancelling waves are still predicted with numerical models (such as that in Fig. 5) in which no negative reflection sites exist.

Alternatively, the initial diastolic BEW could arise from positive reflection of the late systolic FEW. This hypothesis is supported by Fig 7A, which shows data from the two-tube model when initial pressure is uniform throughout the model, but the inlet is connected to a ventricle/valve model to simulate systolic ventricular ejection. As has been observed experimentally, a latesystolic FEW ('a' in Fig. 7A) decreases pressure and leads to valve closure, which produces a brief FCW ('b') associated with the dicrotic notch. This wave complex is partially reflected at 
the junction and returns to the inlet as a BEW ('c') followed by a brief backward compression wave (BCW, 'd'). These waves are then completely reflected at the now-closed valve, producing forward waves 'e' and 'f', which are then repeatedly reflected and re-reflected as in Fig. 6, producing a series of decaying pressure decreases and dicrotic notches. Of course, in the arterial circulation there are many reflection sites. So, if instead of the two-tube model we consider a three-tube model containing two reflection sites (Fig. 7B), a similar phenomenon occurs but the diastolic waves are more numerous, have less intensity and lead to a smoother diastolic pressure decay. In the limit where wave reflection is continuously distributed in space via a tapering tube model (Fig. 7C), the diastolic waves are very small, individual reflections are no longer discernible (i.e. waves are merged) and there is a smooth pressure decay.

\section{In vivo experiments}

\section{Experimental preparation}

Experiments were approved by the institutional animal ethics committee and conformed to guidelines of the National Health and Medical Research Council of Australia. Seven juvenile sheep (weight $21.6 \pm 3.2 \mathrm{~kg}$ ) were studied using similar methods to those described previously

(31). Animals were anesthetized with intramuscular ketamine $(5 \mathrm{mg} / \mathrm{kg})$ and xylazine $(0.1$ $\mathrm{mg} / \mathrm{kg}$ ), followed by $4 \%$ isoflurane delivered by mask. Anesthesia was maintained with isoflurane $(2-3 \%)$, nitrous oxide $(\sim 30 \%)$ and oxygen-enriched air $\left(\mathrm{FiO}_{2} \sim 70 \%\right)$ delivered via a mechanical ventilator, supplemented by an intravenous infusion of ketamine $(1-1.5 \mathrm{mg} / \mathrm{kg} / \mathrm{hr})$ and midazolam $(0.1-0.15 \mathrm{mg} / \mathrm{kg} / \mathrm{hr})$. Ventilation was adjusted to maintain arterial $\mathrm{O}_{2}$ tension at $100-120 \mathrm{mmHg}$ and arterial $\mathrm{CO}_{2}$ at $35-40 \mathrm{mmHg}$. High fidelity central pressure was measured with a 3.5-Fr micromanometer-tipped catheter (SPR-524, Millar Instruments, Houston, TX), inserted via a vascular sheath in the left common carotid artery, and passed into the ascending aorta. Fluid-filled catheters were inserted into the main pulmonary artery and left atrium through a left thoracotomy and, together with the aortic sheath, connected to external pressure sensors, 
with aortic cannula pressure used to calibrate the offset of the corresponding micromanometer pressure. A transit-time flow probe (18-20 mm diameter, Transonics Systems, Ithaca, NY) was placed around the ascending aorta to measure high-fidelity flow.

\section{Experimental protocol}

Baseline variables were recorded after completion of surgery, including a long diastole associated with a ventricular ectopic beat produced by a brisk, light tap with forceps to the right ventricular infundibulum. Hemodynamics were then altered by four sequential interventions in all animals, namely 1) an intravenous infusion of adenosine $(150 \mu \mathrm{g} / \mathrm{kg} / \mathrm{min})$ to lower mean aortic blood pressure by $\sim 20 \mathrm{mmHg}, 2$ ) an intravenous infusion of sodium nitroprusside (SNP, 1$3 \mu \mathrm{g} / \mathrm{kg} / \mathrm{min}$ ) to reduce mean aortic pressure by $\sim 20 \mathrm{mmHg}, 3)$ partial occlusion of the descending thoracic aorta with an adjustable snare to raise mean aortic pressure by $\sim 25 \mathrm{mmHg}$, and 4) an intravenous infusion of phenylephrine (PE, 1.3-3 $\mu \mathrm{g} / \mathrm{kg} / \mathrm{min}$ ) to increase mean aortic pressure by $\sim 25 \mathrm{mmHg}$. Data recording was repeated once hemodynamics had stabilized during each intervention. Subsequently, the drug infusion was stopped or aortic constriction released, and hemodynamics allowed to return to baseline. Baseline data (including a ventricular ectopic with a long diastole) was then recorded again before proceeding with the next intervention. At the end of the study, animals were euthanased with an overdose of sodium pentobarbitone (100 $\mathrm{mg} / \mathrm{kg}$ ) delivered via the left atrial catheter. Blood pressures were recorded continuously during euthanasia, with the final aortic pressure plateau taken as an estimate of mean circulatory pressure $\left(P_{\mathrm{mc}}\right)$.

Results

Hemodynamic data at baseline and during drug infusions are presented in Table 1. Compared with the immediately preceding baseline, mean aortic pressure decreased by $24 \%$ and $29 \%$ after infusion of adenosine and SNP respectively, and increased by $33 \%$ with PE (all $P<0.001$ ); SVR followed a similar pattern. On the other hand, cardiac output increased with adenosine $(P<$ 
0.02), decreased with SNP $(P<0.002)$ and did not change with PE $(P=0.7)$. Arterial compliance, calculated via the pulse pressure method (46), was unchanged with adenosine and SNP but decreased by $26 \%$ with PE $(P<0.002)$. On the other hand, ascending aortic $Z_{\mathrm{c}}$ increased with adenosine and SNP, but did not change with PE.

Pressure and flow integration constants for wave separation, which signify end-diastolic wave potential, decreased by $37 \%$ and $45 \%$ respectively with both adenosine and SNP, but increased by $40 \%$ and $44 \%$ with PE (Table 1$)$. These constants were obtained by setting $P_{\text {ud }}$ to $P_{\mathrm{mc}}(12.4 \pm$ $1.1 \mathrm{mmHg}$ ) measured after euthansia (as in Fig. 2). The equilibrium aortic pressure was reached an average of $15 \pm 2$ seconds after pressure began to drop, and was not different to the corresponding pulmonary arterial and left atrial pressures (mean difference of $0.7 \pm 1.5 \mathrm{mmHg}$ ). To investigate whether $P_{\mathrm{mc}}$ could be estimated without euthanasia, the parameter $P^{\infty}$ described by Wang et al (49) was calculated as the pressure asymptote of an exponential curve fitted to the diastolic pressure decay of an ectopic beat. Taking the average of four baseline recordings in each animal, $P^{\infty}$ was $50.9 \pm 10 \mathrm{mmHg}$, or $38.0 \pm 10.7 \mathrm{mmHg}$ higher than $P_{\text {ud }}(P<0.001)$.

Evidence for a link between the late-systolic FEW and the diastolic pressure decay is shown in Fig. 8, which demonstrates a highly linear relationship $\left(R^{2}=0.67, P<0.001\right)$ between FEW area (i.e. integrated wave intensity, or 'cumulative intensity') and the diastolic pressure decay rate. Here, all baseline and drug infusion data points have been pooled, and the decay rate was calculated as the slope of the line connecting pressures at the start and end of diastole. By contrast, the correlation between early-systolic FCW area and the diastolic pressure decay rate was very weak $\left(R^{2}=0.08, P=0.07\right)$.

Partial occlusion of the descending thoracic aorta to produce a discrete reflection site provided additional data supporting a link between repeated FEW reflection and the diastolic pressure decay. Using ensemble averaged data, in all animals the late systolic FEW $\left(\mathrm{FEW}_{0}\right)$ was followed by a succession of decaying BEWs and FEWs (designated $\mathrm{BEW}_{1}, \mathrm{FEW}_{1}, \mathrm{BEW}_{2}, \mathrm{FEW}_{2}$ ) that caused pressure to oscillate around an exponential curve (Fig. 9). Neither the magnitudes of 
$\mathrm{BEW}_{1}$ and $\mathrm{FEW}_{1}$, nor of $\mathrm{BEW}_{2}$ and $\mathrm{FEW}_{2}$, differed significantly, consistent with the proposition that the forward waves were complete reflections of the respective backward waves (Fig. 10).

\section{Discussion}

This paper has proposed a wave-based (i.e. one-dimensional) paradigm for interpreting arterial diastolic hemodynamics, enabling a complete wave-based explanation of arterial pressure/flow waveforms throughout the cardiac cycle. A key aspect of this paradigm is that the integration constants for calculating the forward and backward (wave) components of pressure and flow are not considered arbitrary. Although this refinement of wave separation theory does not affect the calculation of reflection coefficients or wave intensity (as both are calculated from differences in the wave components), four specific insights follow from it. First, the absolute offsets of the pressure/flow wave components represent pressure/flow wave potential and therefore have a physical meaning. Second, wave potential is intimately linked with reservoir function, with greater wave potential implying greater volume storage in the reservoir. Third, based on analysis of a '1D windkessel', a complete wave-based explanation of self-cancelling diastolic waves has been provided for the first time, with strong support from experimental data. Finally, the 1D windkessel reduces to the classical 0D 2-element windkessel; hence reservoir phenomena are a subset of, and are not distinct from, wave phenomena.

\section{Wave potential and undisturbed pressure}

Blood pressure is a form of potential energy that is converted to kinetic energy where pressure gradients exist. Similarly, a difference in wave potential produces pressure/flow waves (or 'wavefronts') that propagate in compliant arteries. Use of non-arbitrary integration constants for wave separation analysis therefore broadens its explanatory scope to encompass not only information about the passage of waves, but also the potential for waves to be generated. From Equations 8 and 9, the amount of wave potential at any point in space and time depends on four factors, 1) blood pressure, 2) blood flow, 3) characteristic impedance (vessel size and stiffness) 
and 4) undisturbed pressure. Hence, wave potential can be manipulated through the cardiovascular properties (e.g. cardiac function, arterial resistance and compliance) that govern these factors, as demonstrated by simulations (Fig. 5) and with pharmacological agents in vivo (Table 1).

Undisturbed pressure $\left(P_{\mathrm{ud}}\right)$ is essential to the idea of wave potential (19). Although $P_{\mathrm{ud}}$ has implicitly been assigned to mean or end-diastolic pressure in the past, these choices lack a physical basis. Here, we have proposed that the most physiologically justifiable value for $P_{\text {ud }}$ is mean circulatory pressure $\left(P_{\mathrm{mc}}\right)$, the equilibrium pressure that would exist if the heart stopped and blood volume were redistributed to eliminate all pressure differences $(40) . P_{\mathrm{mc}}$ is readily measurable in intact animal studies, although reflex vasoconstriction and the Starling resistor mechanism are potential confounders (40). In our study, reflex vasoconstriction was unlikely to have occurred due to our use of sodium pentobarbitone as the euthanazing agent (7). Similarly, although the Starling resistor mechanism may lead to higher plateau pressures in arteries than veins, left atrial and pulmonary arterial pressures fell to the same plateau as systemic arterial pressure, suggesting that circulatory equilibrium was achieved. Importantly, the $P_{\mathrm{mc}}$ value found in this study $(12.4 \mathrm{mmHg})$ was within the range of mean systemic filling pressure $(10.2 \pm 3.5$ $\mathrm{mmHg}$ ) measured after induction of ventricular fibrillation in humans undergoing implantation of a cardioverter-defibrillator device (13).

A possible alternative to $P_{\mathrm{mc}}$ would be $P^{\infty}$, the pressure constant in the reservoir-wave approach that is calculated in steady-state or ectopic beats (49). However, our analysis showed that $P^{\infty}(50.9 \mathrm{mmHg})$ was $\sim 3$-fold greater than $P_{\mathrm{mc}}(12.4 \mathrm{mmHg})$ and its theoretical limit of 16$18 \mathrm{mmHg}(8) ; P^{\infty}$ and $P_{\mathrm{mc}}$ are therefore not interchangeable. In situations where $P_{\mathrm{mc}}$ cannot be practicably measured, we therefore suggest assuming a value of $11 \mathrm{mmHg}$, noting that reported 
values in humans, i.e. $10.2 \pm 3.5 \mathrm{mmHg}(13)$ and $12.0 \pm 5.4 \mathrm{mmHg}(42)$, have a relatively small variability.

\section{The one-dimensional windkessel}

The windkessel model described by Otto Frank (41) consists of a lumped reservoir compliance and outlet resistance. Since wave effects are neglected, this model does not satisfactorily explain the shape of the systolic pressure/flow waveforms. This problem has been addressed in the past by approximating wave effects via the addition of more lumped elements to the windkessel model, e.g. the 3-element or 4 -element windkessel $(47,50)$, or by separating pressure into 0D reservoir and 1D wave-related components (49). The former approach does not account for wave delays or the spatial evolution of the pressure/flow pulses, while the second approach suffers from conceptual inconsistencies, with the use of hybrid dimensionality leading to errors and physically implausible phenomena when wave analysis is performed $(24,31)$.

In the current study, a '1D windkessel' has been described that contains no lumped $0 \mathrm{D}$ elements. The model in Fig. 6 consisted of two equivalent vessels, one large vessel representing the conduit arteries, and one small vessel representing the peripheral resistance vessels. While this model is useful for investigating basic physical concepts related to wave separation, we do not suggest that this two-segment model is a good model of the arterial system per se. As is now widely recognized, the uniform tube model (which is essentially equivalent to the model in Fig. 6) does not account for the distributed nature of wave reflection in the arterial system (43). Indeed, as was highlighted in Fig. 7, spatially distributed wave reflection produced by a tapering tube model leads to more realistic systolic and diastolic pressure/flow waveforms than models containing one or two discrete reflection sites. However, the term ' $1 \mathrm{D}$ windkessel' can be applied to any 1D model (and indeed, the actual arterial network), which serves as a reminder that these models exhibit and can explain reservoir filling and discharge phenomena. 


\section{Self-cancelling diastolic waves}

Self-cancelling diastolic waves have been a subject of much controversy in recent years. Some have considered these waves to be an artefact of conventional wave separation (5), and eradicating this feature from the wave components was a primary driver of the reservoir-wave paradigm (49). Indeed, interpreting the physiological meaning of $Q_{ \pm}$after a long period of diastole (using the conventional approach with arbitrary integration constants, e.g. Fig. 2A) is problematic. On the other hand, we have previously argued that the $0 \mathrm{D}$ windkessel model is a mathematical subset of the 1D model, and therefore whatever can be explained by the 0D model must also be explicable in terms of the $1 \mathrm{D}$ model $(30,31)$. Although this principle has been elegantly demonstrated in the past $(21,39)$, a comprehensive wave-based explanation of selfcancelling diastolic expansion waves has not previously been advanced.

Our numerical experiments suggested that self-cancelling diastolic waves arise from diffuse and repeated reflection of the late systolic FEW. Although it is well-established that the initial systolic FCW undergoes partial reflection in the vasculature, the literature is surprisingly silent regarding reflection of the late systolic FEW. Nevertheless, an early-diastolic BEW, most likely arising from reflection of the late systolic FEW, is clearly present in many published figures (4, $6,10,15,37,45)$. Our in vivo data supported this hypothesis, with a close relationship between diastolic pressure decay rate and FEW area (Fig. 8), and repeated FEW reflection being clearly evident in the setting of aortic constriction (Fig. 9). A difficulty in discerning repeated reflection of the FEW under normal circumstances probably arises from three main factors. First, arterial wave reflection is diffuse rather than discrete, which causes smoothing and merging of reflected waves (Fig. 7). Second, viscous damping attenuates expansion wave energy (23). Third, nonlinear effects arising from a pressure-dependent wave speed spread out (i.e. attenuate the intensity of) propagating expansion waves (23). Finally, we note that diastolic flow waves are strictly 'self-cancelling' only when flow is equal to zero (i.e. near the aortic valve). Equation 11 
shows that in progressively more peripheral sites, where diastolic run-off occurs, the magnitude of $Q_{+}$will be greater than the magnitude of $Q_{-}$.

The long and short of 'waves'

In 1938, Hamilton and Dow (9) showed that pressure differences in the aorta are "scarcely appreciable" at end-diastole, because "the various waves have died down", a view restated by Wang et al (49). However, Alastruey (1) found that wave reflection from the periphery is primarily responsible for the reservoir pressure. Reservoir phenomena arise because, due to the tree-like arterial structure and associated wave trapping and dispersion, the vascular network acts mainly as a low frequency reflector but high frequency absorber, a principle quantified in the reflection coefficient spectrum (51). Following Lighthill (19), our definition of waves therefore incorporates both 'short' waves, whose propagation in space can be clearly discerned (as can surface waves on water), and 'long' waves whose propagation cannot be discerned because their wavelength is large compared with the dimensions of the system (as with tidal waves). Hence, 0D models such as the windkessel capture the effects of long waves, whereas 1D models capture the effects of both short and long waves.

\section{A wave-only paradigm of arterial hemodynamics}

With incorporation of a wave-based explanation of diastolic phenomena, a wave-only description of arterial hemodynamics throughout the cardiac cycle may now be described. When the ventricle begins to contract, its pressure rises above aortic pressure and opens the aortic valve. The higher ventricular than aortic wave potential induces a FCW that propagates into the aorta, increasing its pressure, flow and wave potential. If no wave reflection occurs, then $\left(P-P_{0}\right) / Z_{\mathrm{c}}=Q-Q_{0}$ (i.e. pressure and flow waveforms have the same shape). However, if reflected compression waves do arise, then $\left(P-P_{0}\right) / Z_{c}>Q-Q_{0}$, causing a further build-up of wave potential in the arterial network. 
Towards the end of systole, ventricular relaxation reduces pressure and flow, generating a FEW (36), and induces closure of the aortic valve. The FEW propagates into the arterial tree and is partially reflected, giving rise to a pressure-decreasing BEW. This wave (or more precisely, the aggregate of diffuse reflection) is reflected and re-reflected (as well as dispersed and attenuated) throughout the arterial tree, with any portion of the BEW arriving back at the aortic valve being completely reflected at the now-closed aortic valve. The resulting FEW component therefore has the same magnitude as the BEW component (see Figs. 9 and 10); both decrease pressure but their flow effects cancel (self-cancelling waves). Due to the diffuse nature of the reflections, the initial 'short' waves are rapidly transformed into 'long' waves that cause pressure to decay almost uniformly throughout the system (49). Wave reflection in the forward direction is incomplete, however, so wave potential progressively leaks from large to small vessels, thereby giving rise to the quasi-exponential pressure decay and discharge of the arterial reservoir.

\section{Non-linearities in the arterial system}

The analysis presented in this paper has assumed a constant $Z_{c}$ and linear addition of forward and backward components. In reality however, $c$ and $A$ (and therefore $Z_{c}$ ) are pressuredependent and wave components do not add linearly. Using the method of characteristics, these non-linearities can be accounted for in wave separation analysis if the pressure-area relation $P(A)$ is known $(26,38)$, with characteristic variables $\left(W_{+}\right.$and $\left.W_{-}\right)$separately governing the forward and backward waves. To perform non-linear wave separation, expressions of the form $d P_{ \pm}=f\left(d W_{ \pm}\right)$are integrated. As with $P_{ \pm}$and $Q_{ \pm}$, the integration constants, and therefore absolute values, of $W_{ \pm}$have previously been considered arbitrary. However, the principles advanced in this paper suggest that $P_{\text {ud }}$ should be used for the integration constants in this analysis also, with $W_{ \pm}\left(P_{\text {ud }}\right)=0$. 


\section{Conclusion}

Physiologically meaningful values of the integration constants in wave separation analysis can be obtained by identifying undisturbed pressure as mean circulatory pressure. The resulting absolute values of the wave components represent 'wave potential', gradients of which produce propagating pressure/flow wavefronts. Repeated and diffuse reflection of the late systolic FEW along with leakage of wave potential in a ' $1 \mathrm{D}$ windkessel' during diastole produce selfcancelling expansion waves, a quasi-exponential pressure decay and discharge of the arterial reservoir. These wave-based concepts of diastolic hemodynamics permit a comprehensive wavebased paradigm of arterial hemodynamics throughout the cardiac cycle.

\section{Acknowledgments}

We thank Magdy Sourial and Dr. Kelly Kenna for assistance with experimental studies. This work was supported in part by the Victorian Government's Operational Infrastructure Support Program. J.P. Mynard is supported by a CJ Martin Early Career Fellowship from the National Health and Medical Research Council of Australia.

\section{Conflicts of Interest}

None.

\section{References}

1. Alastruey J. On the Mechanics Underlying the Reservoir-Excess Separation in Systemic Arteries and their Implications for Pulse Wave Analysis. Cardiovasc Eng 10: 176-189, 2010.

2. Alastruey J, Parker K, Peiró J, and Sherwin S. Analysing the pattern of pulse waves in arterial networks: a time-domain study. Journal of Engineering Mathematics 64: 331-351, 2009.

3. Berger DS, Li JK, and Noordergraaf A. Differential effects of wave reflections and peripheral resistance on aortic blood pressure: a model-based study. Am J Physiol Heart Circ Physiol 266: H1626-1642, 1994. 
4. Davies JE, Alastruey J, Francis DP, Hadjiloizou N, Whinnett ZI, Manisty CH, AguadoSierra J, Willson K, Foale RA, Malik IS, Hughes AD, Parker KH, and Mayet J. Attenuation of Wave Reflection by Wave Entrapment Creates a "Horizon Effect" in the Human Aorta. Hypertension 60: 778-785, 2012.

5. Davies JE, Hadjiloizou N, Leibovich D, Malaweera A, Alastruey-Arimon J, Whinnett ZI, Manisty CH, Francis DP, Aguado-Sierra J, Foale RA, Malik IS, Parker KH, Mayet J, and Hughes AD. Importance of the aortic reservoir in determining the shape of the arterial pressure waveform - The forgotten lessons of Frank. Artery Res 1: 40-45, 2007.

6. Grant DA, Hollander E, Skuza EM, and Fauchere JC. Interactions between the right ventricle and pulmonary vasculature in the fetus. J Appl Physiol 87: 1637-1643, 1999.

7. Green J. Pressure-flow and volume-flow relationships of the systemic circulation of the dog. Am J Physiol 229: 761-769, 1975.

8. Guyton AC, Polizo D, and Armstrong GG. Mean Circulatory Filling Pressure Measured Immediately After Cessation of Heart Pumping. Am J Physiol 179: 261-267, 1954.

9. Hamilton WF and Dow P. AN EXPERIMENTAL STUDY OF THE STANDING WAVES IN THE PULSE PROPAGATED THROUGH THE AORTA. Am J Physiol 125: 48-59, 1938.

10. Hollander EH, Wang JJ, Dobson GM, Parker KH, and Tyberg JV. Negative wave reflections in pulmonary arteries. Am J Physiol Heart Circ Physiol 281: H895-902, 2001.

11. Hughes A and Parker K. Forward and backward waves in the arterial system: impedance or wave intensity analysis? Med Biol Eng Comput 47: 207-210, 2009.

12. Hughes AD, Davies J, and Parker KH. The importance of wave reflection: a comparison of wave intensity analysis and separation of pressure into forward and backward components. 35th Annual International Conference of the IEEE Engineering in Medicine and Biology Society, Osaka, Japan, 2013, p. 229-232. 
13. Jellinek H, Krenn H, Oczenski W, Veit F, Schwarz S, and Fitzgerald RD. Influence of positive airway pressure on the pressure gradient for venous return in humans. J Appl Physiol 88: 926-932, 2000.

14. Jones CJ, Sugawara M, Kondoh Y, Uchida K, and Parker KH. Compression and expansion wavefront travel in canine ascending aortic flow: wave intensity analysis. Heart Vessels 16: 91-98, 2002.

15. Khir AW and Parker KH. Wave intensity in the ascending aorta: effects of arterial occlusion. J Biomech 38: 647-655, 2005.

16. Laskey WK and Kussmaul WG. Arterial wave reflection in heart failure. Circulation 75: $711-722,1987$.

17. Laxminarayan S. The calculation of forward and backward waves in the arterial system. Med Biol Eng Comput 17: 130-130, 1979.

18. Li JK. Time domain resolution of forward and reflected waves in the aorta. IEEE Trans Biomed Eng 33: 783-785, 1986.

19. Lighthill J. Waves in Fluids. London: Cambridge University, 1978.

20. Manisty CH, Zambanini A, Parker KH, Davies JE, Francis DP, Mayet J, McG Thom SA, and Hughes AD. Differences in the Magnitude of Wave Reflection Account for Differential Effects of Amlodipine- Versus Atenolol-Based Regimens on Central Blood Pressure. An AngloScandinavian Cardiac Outcome Trial Substudy. Hypertension 54: 724-730, 2009.

21. Mohiuddin MW, Laine GA, and Quick CM. Increase in pulse wavelength causes the systemic arterial tree to degenerate into a classical windkessel. Am J Physiol Heart Circ Physiol 293: H1164-1171, 2007.

22. Murgo JP, Westerhof N, Giolma JP, and Altobelli SA. Manipulation of ascending aortic pressure and flow wave reflections with the Valsalva maneuver: relationship to input impedance. Circulation 63: 122-132, 1981. 
23. Mynard J, Penny DJ, and Smolich JJ. Wave intensity amplification and attenuation in non-linear flow: Implications for the calculation of local reflection coefficients. $J$ Biomech 41: 3314-3321, 2008.

24. Mynard JP. Assessment of conceptual inconsistencies in the hybrid reservoir-wave model. 35th Annual International Conference of the IEEE Engineering in Medicine and Biology Society, 2013, p. 213-216.

25. Mynard JP. Computer modelling and wave intensity analysis of perinatal cardiovascular function and dysfunction (PhD Thesis). Melbourne, Australia: University of Melbourne 2011.

26. Mynard JP, Davidson MR, Penny DJ, and Smolich JJ. Non-linear separation of pressure, velocity and wave intensity into forward and backward components. Med Biol Eng Comput 50: 641-648, 2012.

27. Mynard JP, Davidson MR, Penny DJ, and Smolich JJ. A numerical model of neonatal pulmonary atresia with intact ventricular septum and RV-dependent coronary flow. International Journal for Numerical Methods in Biomedical Engineering 26: 843-861, 2010.

28. Mynard JP, Davidson MR, Penny DJ, and Smolich JJ. A simple, versatile valve model for use in lumped parameter and one-dimensional cardiovascular models. International Journal for Numerical Methods in Biomedical Engineering 28: 626-641, 2012.

29. Mynard JP and Nithiarasu P. A 1D arterial blood flow model incorporating ventricular pressure, aortic valve and regional coronary flow using the locally conservative Galerkin (LCG) method. Communications in Numerical Methods in Engineering 24: 367-417, 2008.

30. Mynard JP, Penny DJ, Davidson MR, and Smolich JJ. The reservoir-wave paradigm. $J$ Hypertens 30: 1881-1883, 2012.

31. Mynard JP, Penny DJ, Davidson MR, and Smolich JJ. The reservoir-wave paradigm introduces error into arterial wave analysis: a computer modelling and in-vivo study. J Hypertens 30: 734-743, 2012. 
32. Nichols WW and O'Rourke MF. McDonald's blood flow in arteries: theoretical, experimental, and clinical principles: CRC Press, 2011.

33. O'Rourke MF and Yaginuma T. Wave reflections and the arterial pulse. Arch Intern Med 144: 366, 1984.

34. Parker KH. An introduction to wave intensity analysis. Med Biol Eng Comput 47: 175188, 2009.

35. Parker KH and Jones CJ. Forward and backward running waves in the arteries: analysis using the method of characteristics. J Biomech Eng 112: 322-326, 1990.

36. Parker KH, Jones CJ, Dawson JR, and Gibson DG. What stops the flow of blood from the heart? Heart Vessels 4: 241-245, 1988.

37. Penny DJ, Mynard JP, and Smolich JJ. Aortic wave intensity analysis of ventricularvascular interaction during incremental dobutamine infusion in adult sheep. Am J Physiol Heart Circ Physiol 294: H481-489, 2008.

38. Pythoud F, Stergiopulos N, and Meister JJ. Forward and backward waves in the arterial system: nonlinear separation using Riemann invariants. Technol Health Care 3: 201-207, 1995.

39. Quick CM, Berger DS, and Noordergraaf A. Apparent arterial compliance. Am $J$ Physiol Heart Circ Physiol 274: H1393-1403, 1998.

40. Rothe CF. Mean circulatory filling pressure: its meaning and measurement. J Appl Physiol 74: 499-509, 1993.

41. Sagawa K, Lie RK, and Schaefer J. Translation of Otto Frank's paper "Die Grundform des Arteriellen Pulses" Zeitschrift fur Biologie 37: 483-526 (1899). J Mol Cell Cardiol 22: 253277, 1990.

42. Schipke JD, Heusch G, Sanii AP, Gams E, and Winter J. Static filling pressure in patients during induced ventricular fibrillation. Am J Physiol Heart Circ Physiol 285: H2510H2515, 2003. 
43. Segers P, Mynard J, Taelman L, Vermeersch S, and Swillens A. Wave reflection: Myth or reality? Artery Res 6: 7-11, 2012.

44. Segers P, Swillens A, and Vermeersch S. Reservations on the reservoir. J Hypertens 30: 676-678, 2012.

45. Smolich JJ, Mynard JP, and Penny DJ. Simultaneous pulmonary trunk and pulmonary arterial wave intensity analysis in fetal lambs: evidence for cyclical, midsystolic pulmonary vasoconstriction. American Journal of Physiology - Regulatory, Integrative \& Comparative Physiology 294: R1554-1562, 2008.

46. Stergiopulos N, Meister J, and Westerhof N. Simple and accurate way for estimating total and segmental arterial compliance: The pulse pressure method. Ann Biomed Eng 22: 392$397,1994$.

47. Stergiopulos N, Westerhof BE, and Westerhof N. Total arterial inertance as the fourth element of the windkessel model. Am J Physiol Heart Circ Physiol 276: H81-H88, 1999.

48. Vermeersch S, Rietzschel E, De Buyzere M, Van Bortel L, Gillebert T, Verdonck P, and Segers P. The reservoir pressure concept: the 3-element windkessel model revisited? Application to the Asklepios population study. Journal of Engineering Mathematics 64: 417428, 2009.

49. Wang JJ, O'Brien AB, Shrive NG, Parker KH, and Tyberg JV. Time-domain representation of ventricular-arterial coupling as a windkessel and wave system. American Journal of Physiology Heart \& Circulatory Physiology 284: H1358-H1368, 2003.

50. Westerhof N, Elzinga G, and Sipkema P. An artificial arterial system for pumping hearts. J Appl Physiol 31: 776-781, 1971.

51. Westerhof N, Sipkema P, van den Bos GC, and Elzinga G. Forward and backward waves in the arterial system. Cardiovasc Res 6: 648-656, 1972. 
52. Westerhof $\mathbf{N}$ and Westerhof BE. Forward and backward pressure waves in the arterial system do not represent reality - Rebuttal from Nico Westerhof and Berend E. Westerhof. The Journal of Physiology 591: 1175, 2013. 
Figure Captions

Fig. 1. Pressure and flow wave separation in a normal beat, followed by a beat with an extended diastole in the sheep ascending aorta. Arrows point to 'self-cancelling' flow waves during diastole.

Fig. 2. Net (black), forward (red) and backward (blue) pressure and flow in sheep ascending aorta associated with euthanasia and subsequent asystole. Integration constants were (A) chosen on the basis of graphing convenience for steady state beats (i.e. arbitrarily), or (B) using values calculated with undisturbed pressure $\left(P_{\mathrm{ud}}\right)$ equal to measured mean circulatory pressure $\left(P_{\mathrm{mc}}\right)$.

Fig. 3. Wave separation for same beats as in Fig. 1 but using non-arbitrary integration constants derived from measured undisturbed pressure $\left(P_{\mathrm{ud}}=13.8 \mathrm{mmHg}\right)$. The absolute values of the pressure and flow wave components signify wave potential, while the pulsatile components arise from pressure/flow waves.

Fig. 4. Numerical experiment in which a barrier between two tubes is suddenly removed (at $t=10 \mathrm{~ms})$. Pressure in tube $1(x<0)$ is higher than in tube $2(x>0)$ before the barrier is removed and pressure-equalizing waves propagate in both directions afterwards. There is a closed end at $x=-15 \mathrm{~cm}$, leading to complete reflection of the backward wave, whereas tube 2 is well-matched (i.e. infinitely long).

Fig. 5. Simulations from the closed-loop circulation model described in (28) with (A) normal baseline parameters, (B) systemic arterial wave speed doubled, (C) peripheral arterial resistance doubled, or (D) peripheral arterial resistance halved.

Fig. 6. Demonstration of a one-dimensional windkessel. At $t=0$ pressure is $80 \mathrm{mmHg}$ in the large tube and $0 \mathrm{mmHg}$ in the small tube. Pressures and flows are plotted at the inlet (black lines), middle (green lines) and outlet (red lines) of the large tube. A succession of forward and backward expansion wavefronts (illustrated in the lower panel and seen as step decreases in pressure) produce a quasi-exponential decay of pressure in the large tube.

Fig. 7. Numerical experiment demonstrating how repeated reflection of the late-systolic forward expansion wave gives rise to the exponential pressure decay. (A) Two-tube model with one 
reflection site, (B) three-tube model with two reflection sites, (C) tapering tube model giving rise to spatially distributed wave reflection. See text for a description of the wave labels a-f.

Fig. 8. Relation between diastolic pressure decay rate and forward expansion wave area in the ascending aorta of sheep $(n=7)$ at baseline and during infusions of adenosine, sodium nitroprusside and phenylephrine.

Fig. 9. (A) Pressure and (B) wave intensity in sheep ascending aorta during mechanical constriction of the descending thoracic aorta to create a discrete reflection site. (C) Zoomed-in plot showing early diastolic pressure, which oscillates around an exponential curve (dicrotic notch is indicated by a grey line), with (D) the corresponding wave intensity profile revealing an underlying series of decaying forward and backward expansion waves $\left(\mathrm{BEW}_{1}, \mathrm{FEW}_{1}, \mathrm{BEW}_{2}\right.$, $\left.\mathrm{FEW}_{2}\right)$ following the late systolic forward expansion wave $\left(\mathrm{FEW}_{0}\right)$. Compression waves are shaded and expansion waves are unshaded.

Fig. 10. Peak wave intensity magnitude of the early diastolic waves in sheep undergoing aortic constriction $(n=7)$. Wave labels are defined in Fig. 9. Data presented as mean $\pm \mathrm{SD}$, NS = no significant difference. 
Table 1. Hemodynamics and integration constants for wave separation in experimental studies.

\begin{tabular}{lcccc}
\hline & Baseline & Adenosine & SNP & PE \\
Heart Rate (beats/min) & $127 \pm 13$ & $129 \pm 17$ & $125 \pm 14$ & $126 \pm 15^{*}$ \\
Mean AoT $P(\mathrm{mmHg})$ & $86.7 \pm 5.9$ & $65.9 \pm 6.7^{\dagger}$ & $64.2 \pm 5.7^{\dagger}$ & $112.2 \pm 10.3^{\dagger}$ \\
SVR $(\mathrm{mmHg} . \mathrm{s} / \mathrm{mL})$ & $1.98 \pm 0.56$ & $1.33 \pm 0.31^{\dagger}$ & $1.64 \pm 0.53^{\dagger}$ & $2.41 \pm 0.74^{\dagger}$ \\
$\mathrm{C}_{\text {art }}(\mathrm{mL} / \mathrm{mmHg})$ & $0.81 \pm 0.17$ & $0.74 \pm 0.09$ & $0.79 \pm 0.12$ & $0.59 \pm 0.15^{\ddagger}$ \\
CO $(\mathrm{L} / \mathrm{min})$ & $2.63 \pm 0.55$ & $2.84 \pm 0.48^{*}$ & $2.31 \pm 0.57^{\dagger}$ & $2.83 \pm 0.66$ \\
AoT $Z_{\mathrm{c}}(\mathrm{mmHg} . \mathrm{s} / \mathrm{mL})$ & $0.14 \pm 0.04$ & $0.17 \pm 0.06^{\dagger}$ & $0.18 \pm 0.06^{*}$ & $0.15 \pm 0.04$ \\
& & & & \\
Integration Constants & & & & \\
AoT $P_{+}^{0}(\mathrm{~L} / \mathrm{min})$ & $31.5 \pm 4.2$ & $19.8 \pm 4.7^{\dagger}$ & $21.0 \pm 4.6^{\dagger}$ & $41.8 \pm 6.1^{\dagger}$ \\
AoT $P_{-}^{0}(\mathrm{~L} / \mathrm{min})$ & $30.9 \pm 4.3$ & $20.6 \pm 4.5^{\dagger}$ & $21.5 \pm 3.9^{\dagger}$ & $40.8 \pm 6.0^{\dagger}$ \\
AoT $Q_{+}^{0}(\mathrm{~L} / \mathrm{min})$ & $14.5 \pm 4.8$ & $7.8 \pm 3.9^{\dagger}$ & $8.2 \pm 4.2^{\dagger}$ & $18.3 \pm 5.7^{\ddagger}$ \\
AoT $Q_{-}^{0}(\mathrm{~L} / \mathrm{min})$ & $-14.3 \pm 4.9$ & $-8.1 \pm 4.9^{\dagger}$ & $-8.3 \pm 4.1^{\dagger}$ & $-17.9 \pm 5.7^{\ddagger}$ \\
\hline
\end{tabular}

Data are expressed as means $\pm \mathrm{SD} ; \mathrm{n}=7$. Abbreviations: AoT, aortic trunk; $\mathrm{C}_{\text {art }}$, total arterial compliance from the pulse pressure method; $\mathrm{CO}$, cardiac output; $P$, pressure; $Z_{\mathrm{c}}$, characteristic impedance. ${ }^{*} P<0.05,{ }^{\dagger} P<0.002,{ }^{\dagger} P \leq 0.001$ compared with immediately preceding baseline (averaged baseline data presented) 
Fig. 1. Pressure and flow wave separation in a normal beat, followed by a beat with an extended diastole in the sheep ascending aorta. Arrows point to 'self-cancelling' flow waves during diastole.
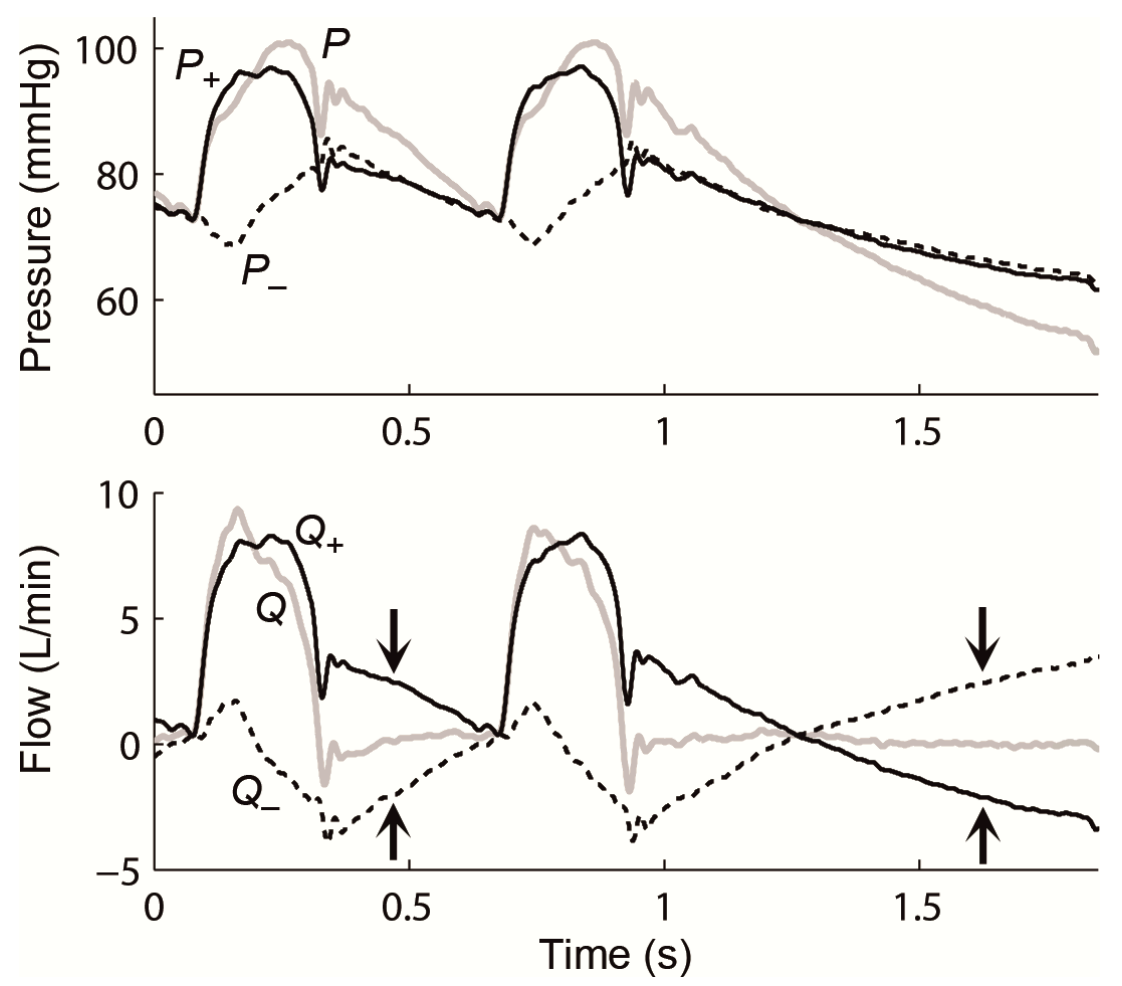
Fig. 2. Net (black), forward (red) and backward (blue) pressure and flow in sheep ascending aorta associated with euthanasia and subsequent asystole. Integration constants were (A) chosen on the basis of graphing convenience for steady state beats (i.e. arbitrarily), or (B) using values calculated with undisturbed pressure $\left(P_{\mathrm{ud}}\right)$ equal to measured mean circulatory pressure $\left(P_{\mathrm{mc}}\right)$.

A

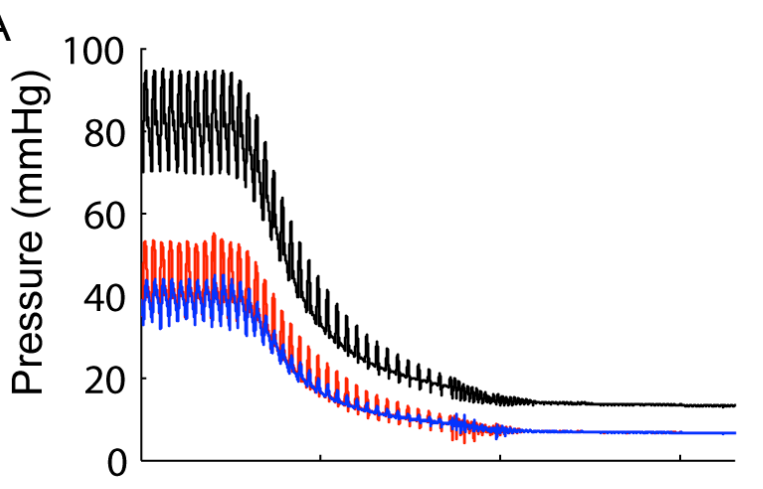

B

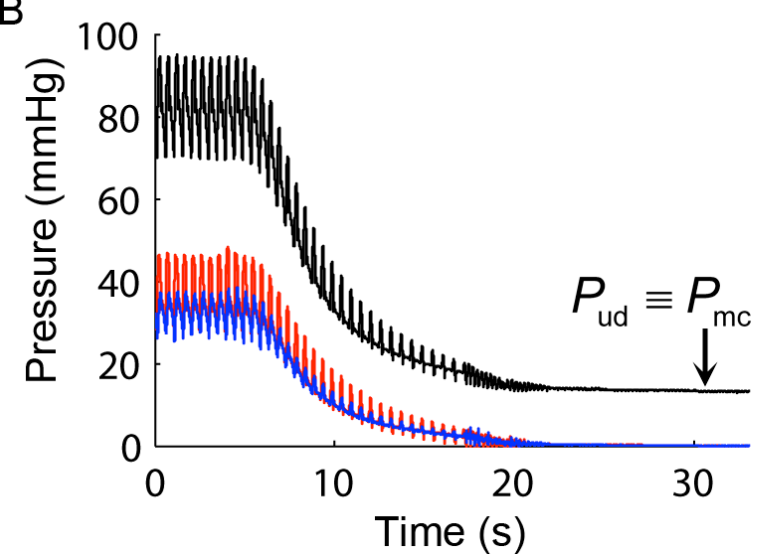

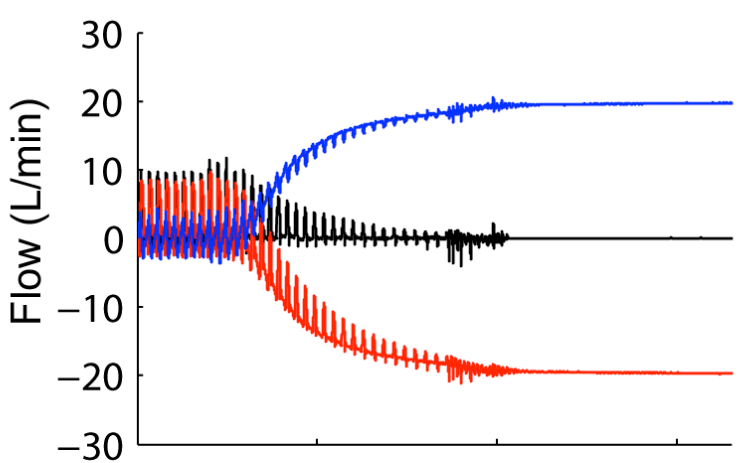

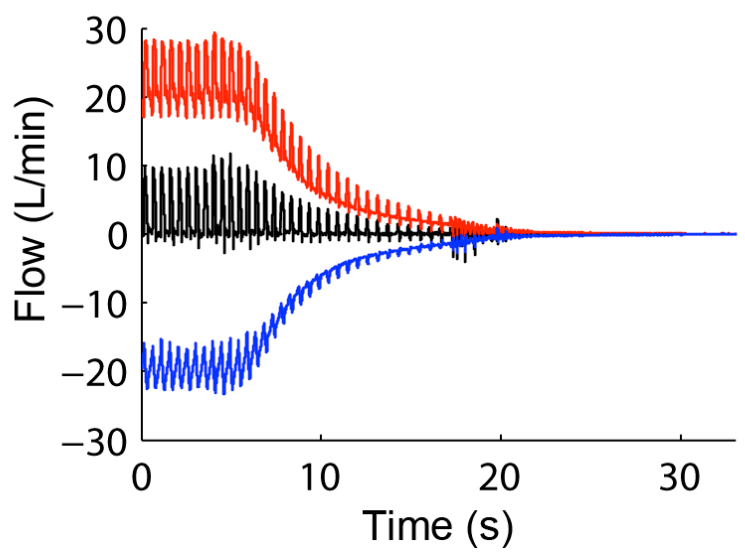


Fig. 3. Wave separation for same beats as in Fig. 1 but using non-arbitrary integration constants derived from measured undisturbed pressure $\left(P_{\mathrm{ud}}=13.8 \mathrm{mmHg}\right)$. The absolute values of the pressure and flow wave components signify wave potential, while the pulsatile components arise from pressure/flow waves.
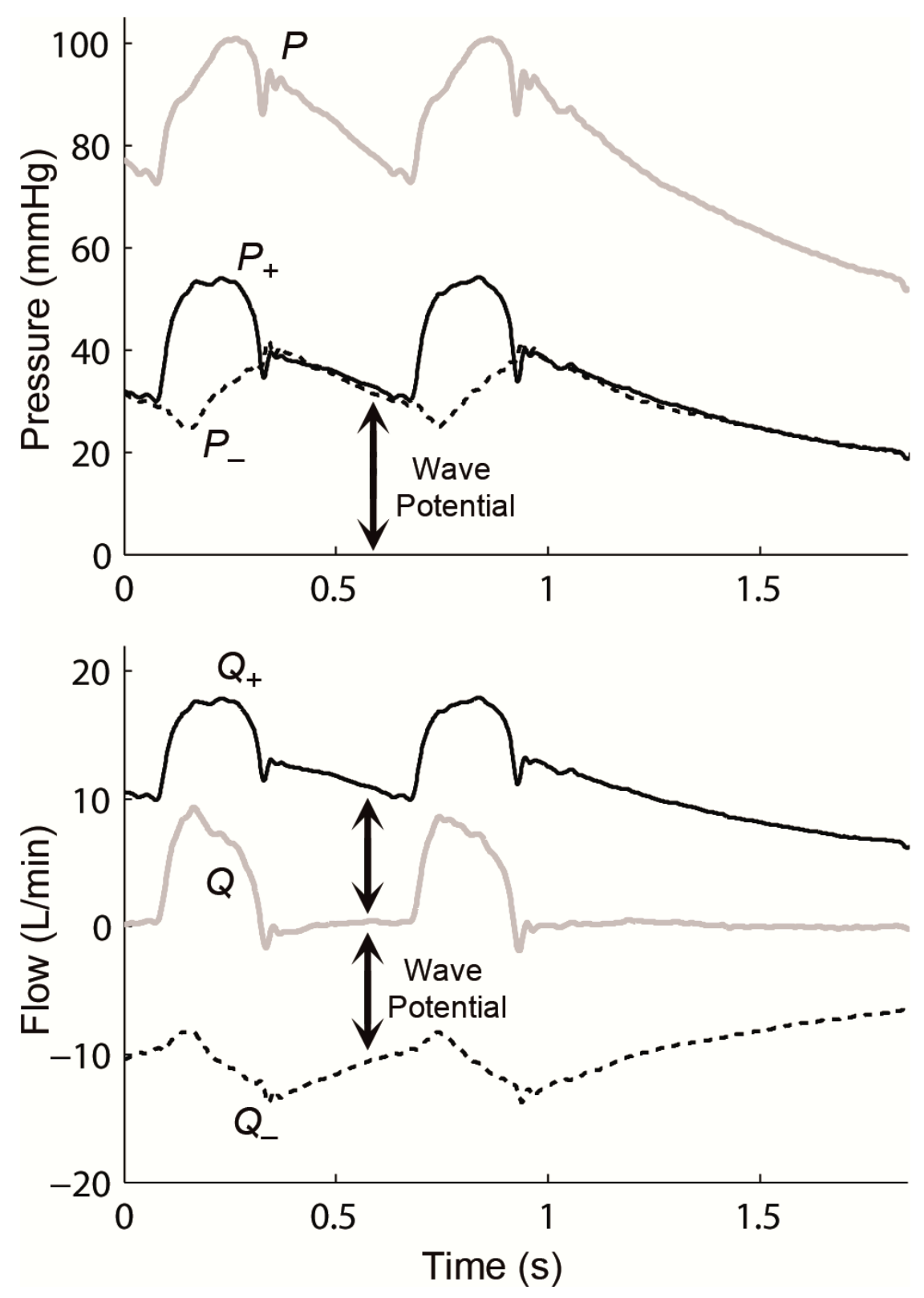
Fig. 4. Numerical experiment in which a barrier between two tubes is suddenly removed (at $t=10 \mathrm{~ms})$. Pressure in tube $1(x<0)$ is higher than in tube $2(x>0)$ before the barrier is removed and pressure-equalizing waves propagate in both directions afterwards. There is a closed end at $x=-15 \mathrm{~cm}$, leading to complete reflection of the backward wave, whereas tube 2 is well-matched (i.e. infinitely long).
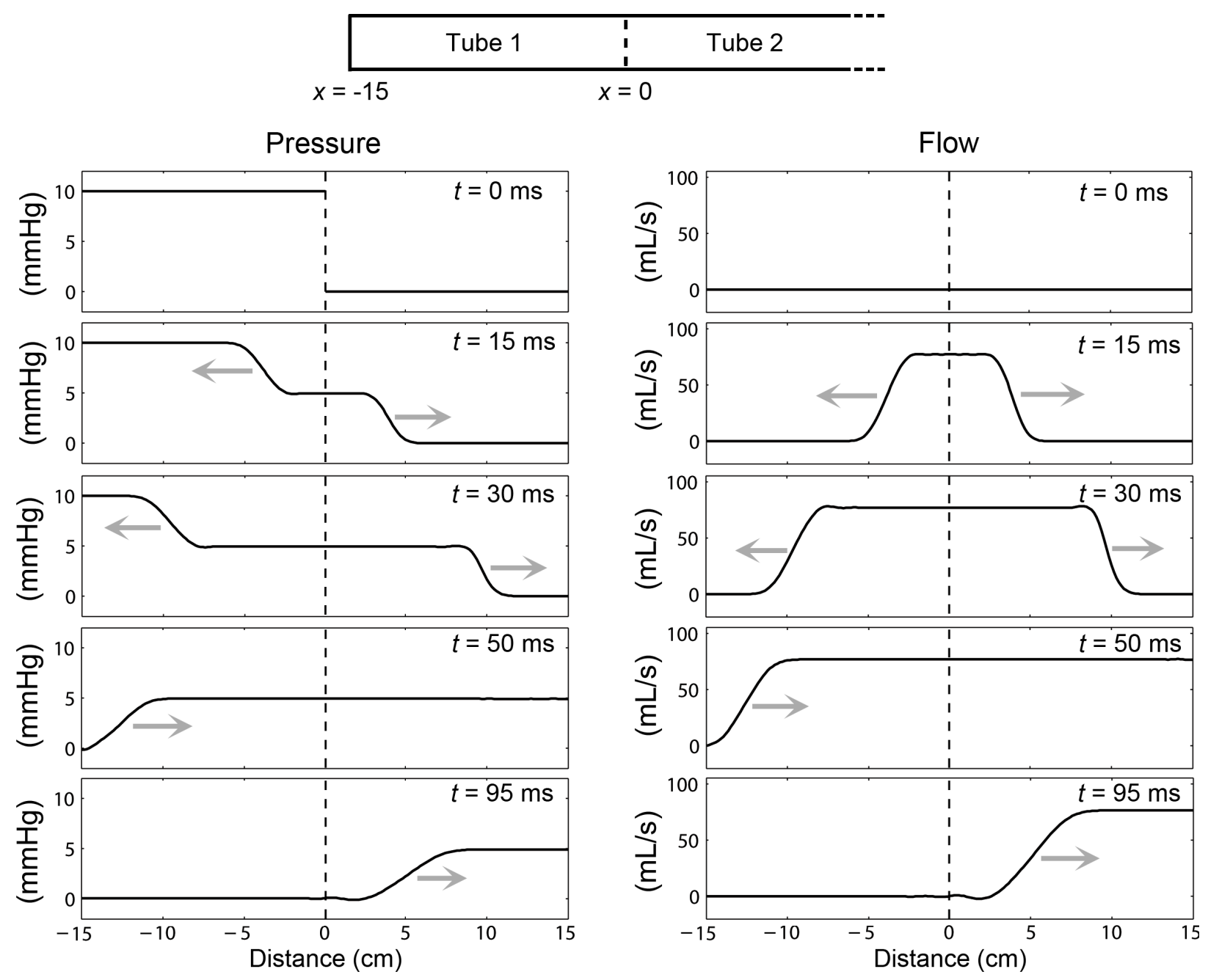
Fig. 5. Simulations from the closed-loop circulation model described in (28) with (A) normal baseline parameters, (B) systemic arterial wave speed doubled, (C) peripheral arterial resistance doubled, or (D) peripheral arterial resistance halved.
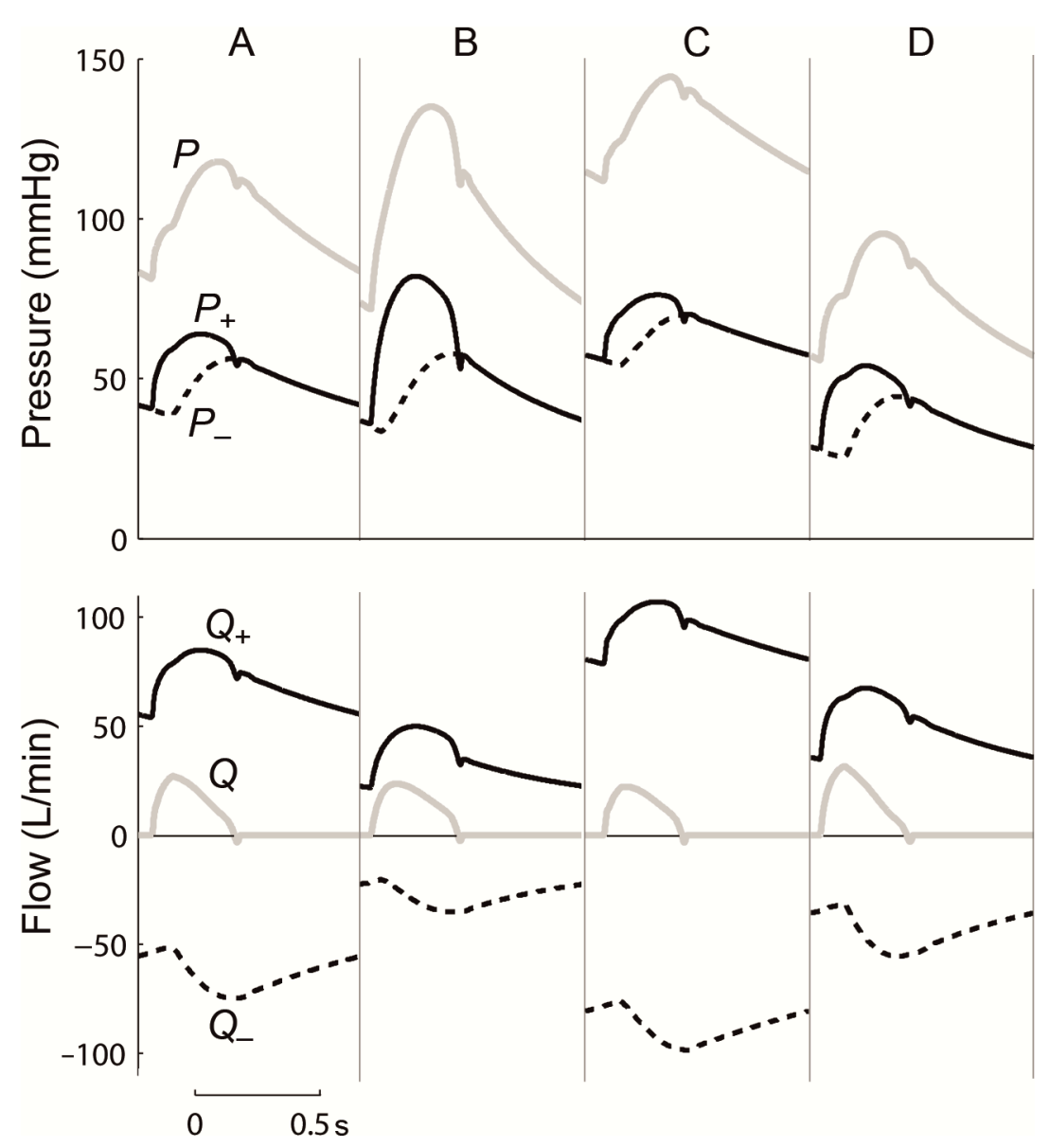
Fig. 6. Demonstration of a one-dimensional windkessel. At $t=0$ pressure is $80 \mathrm{mmHg}$ in the large tube and $0 \mathrm{mmHg}$ in the small tube. Pressures and flows are plotted at the inlet (black lines), middle (green lines) and outlet (red lines) of the large tube. A succession of forward and backward expansion wavefronts (illustrated in the lower panel and seen as step decreases in pressure) produce a quasi-exponential decay of pressure in the large tube.
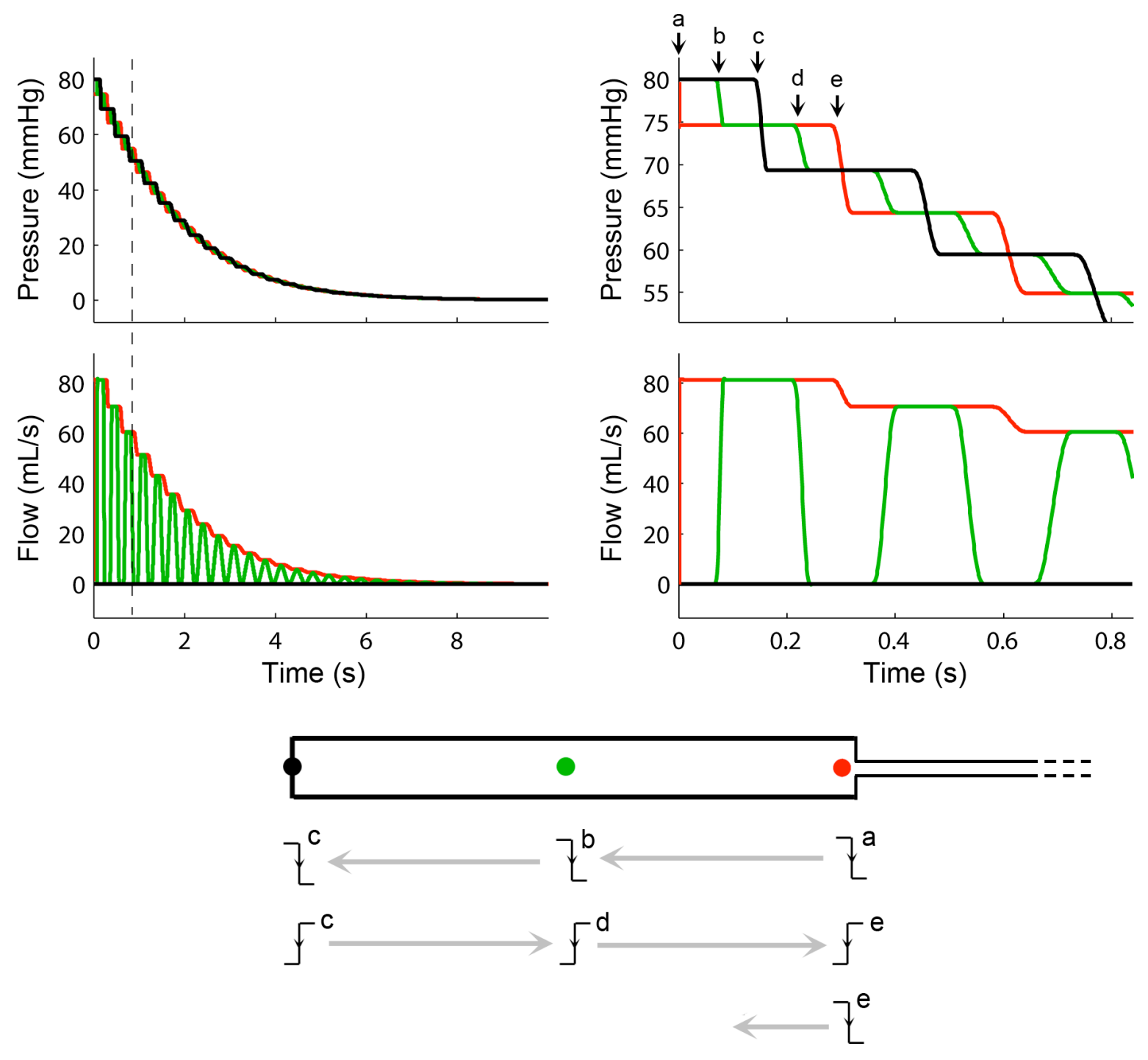
Fig. 7. Numerical experiment demonstrating how repeated reflection of the late-systolic forward expansion wave gives rise to the exponential pressure decay. (A) Two-tube model with one reflection site, (B) three-tube model with two reflection sites, $(\mathrm{C})$ tapering tube model giving rise to spatially distributed wave reflection. See text for a description of the wave labels a-f. Compression and expansion waves are shaded and unshaded respectively; forward and backward waves are red and blue respectively.
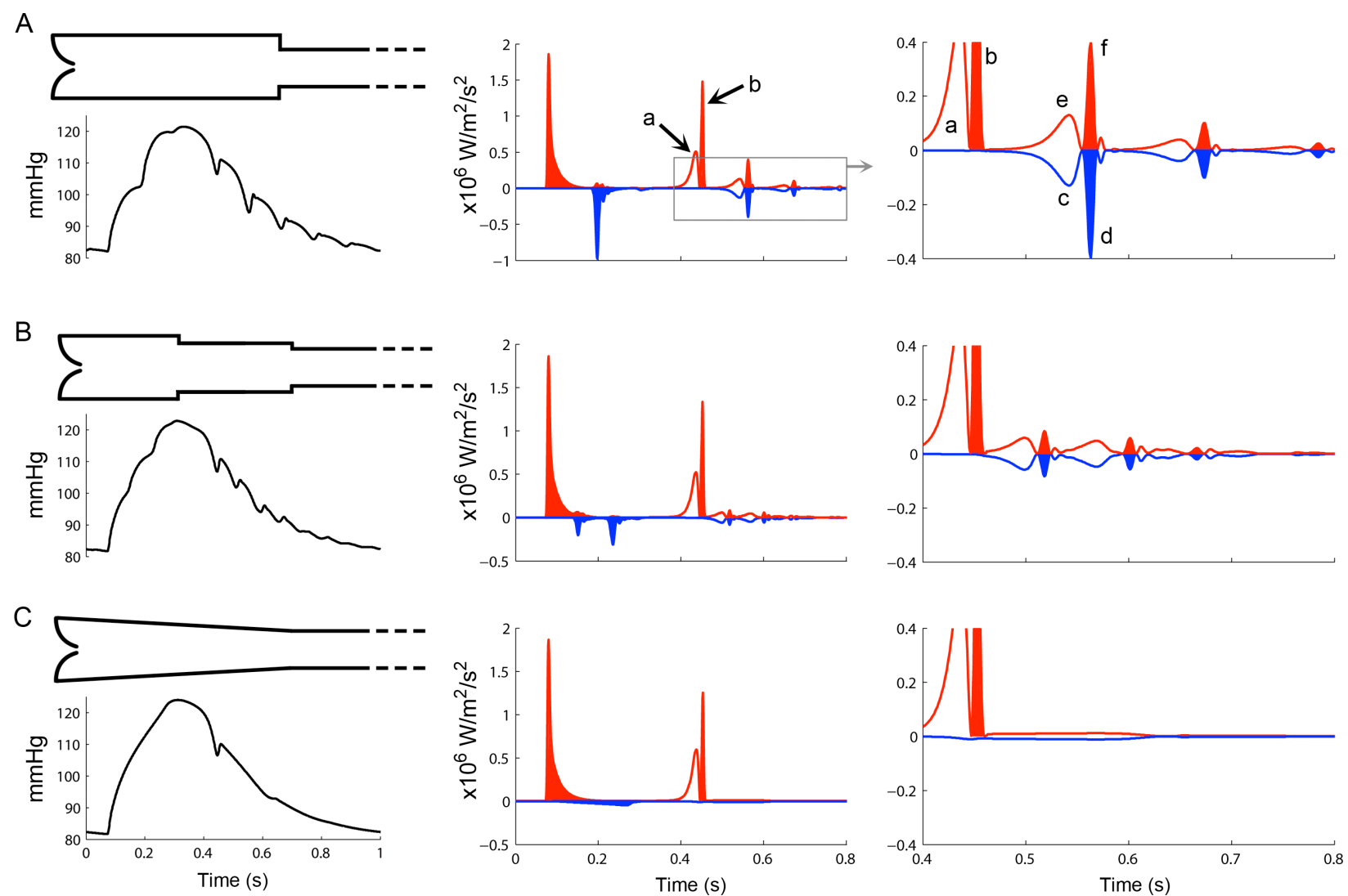
Fig. 8. Relation between diastolic pressure decay rate and forward expansion wave area in the ascending aorta of sheep $(n=7)$ at baseline and during infusions of adenosine, sodium nitroprusside and phenylephrine.

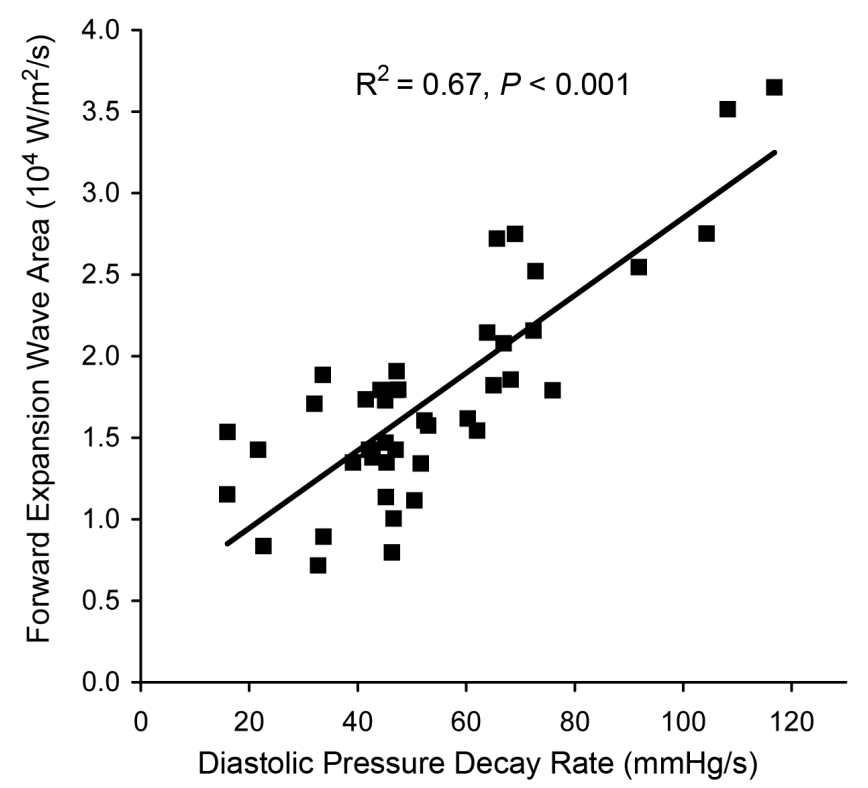


Fig. 9. (A) Pressure and (B) wave intensity in sheep ascending aorta during mechanical constriction of the descending thoracic aorta to create a discrete reflection site. (C) Zoomed-in plot showing early diastolic pressure, which oscillates around an exponential curve (dicrotic notch is indicated by a grey line), with (D) the corresponding wave intensity profile revealing an underlying series of decaying forward and backward expansion waves $\left(\mathrm{BEW}_{1}, \mathrm{FEW}_{1}, \mathrm{BEW}_{2}\right.$, $\mathrm{FEW}_{2}$ ) following the late systolic forward expansion wave $\left(\mathrm{FEW}_{0}\right)$. Compression waves are shaded and expansion waves are unshaded; forward and backward waves are red and blue lines respectively.
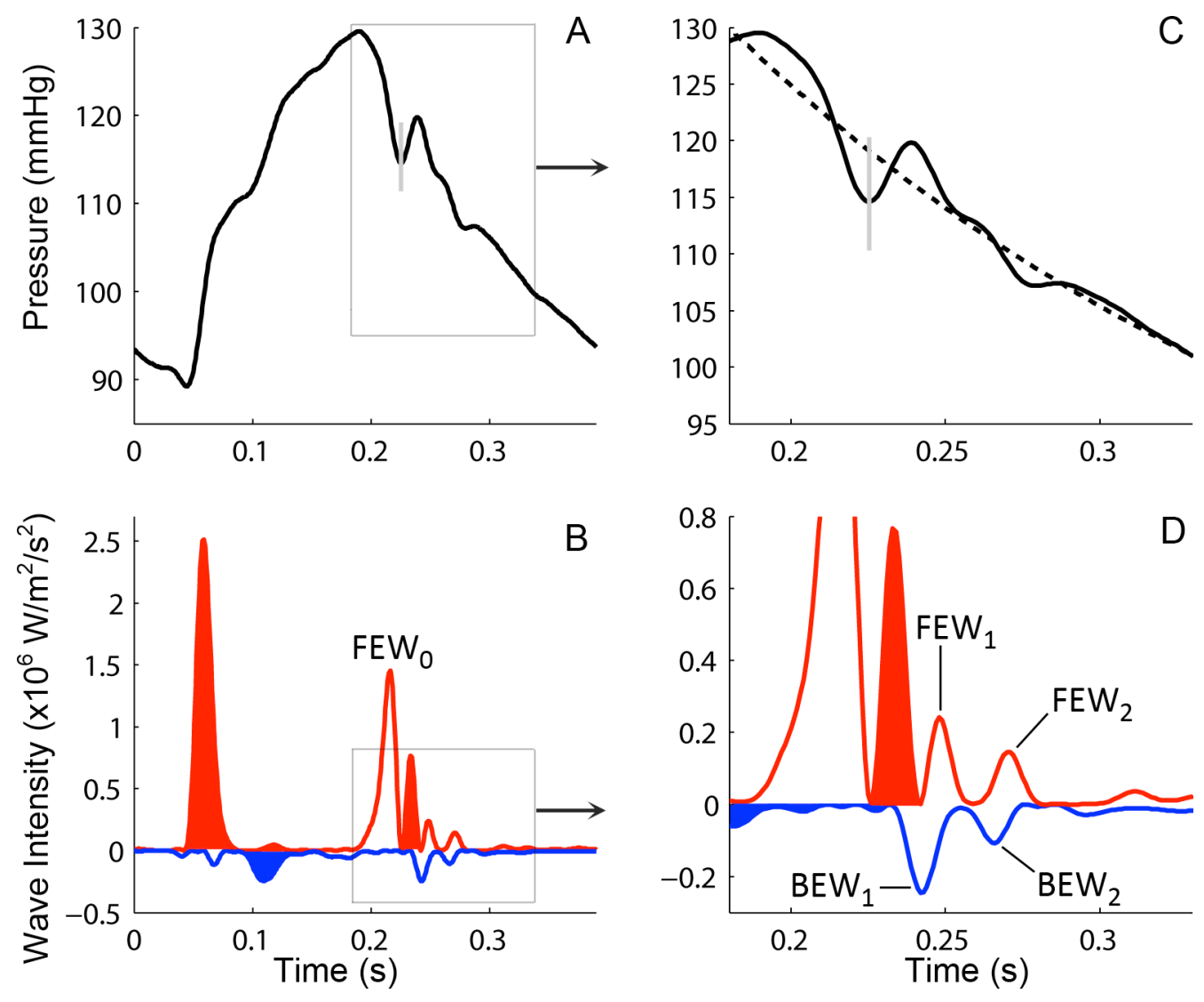
Fig. 10. Peak wave intensity magnitude of the early diastolic waves in sheep undergoing aortic constriction $(n=7)$. Wave labels are defined in Fig. 9. Data presented as mean \pm SD, NS $=$ no significant difference.

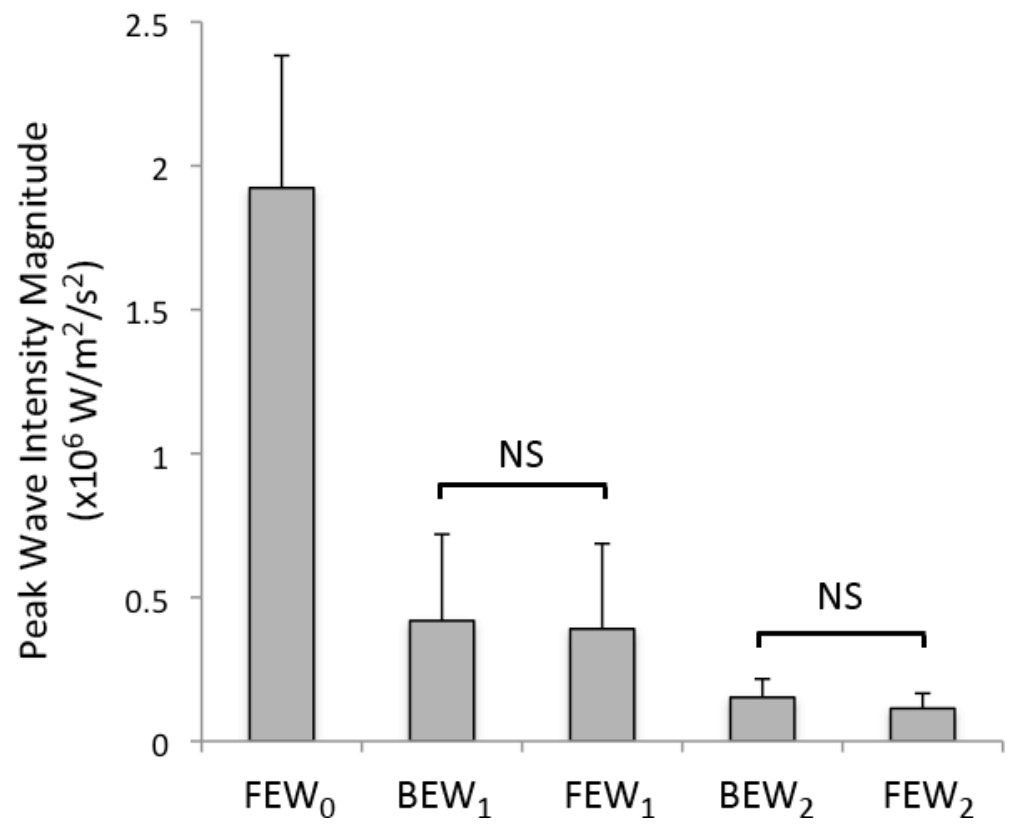

